\title{
Investigation of transport models in ASDEX Upgrade current ramps
}

\author{
S. Fietz, E. Fable, J. Hobirk, R. Fischer, C. Fuchs, G. \\ Pereverzev†, F. Ryter and the ASDEX Upgrade Team \\ Max Planck Institute for Plasma Physics, EURATOM Association, Garching, \\ Germany \\ $\dagger$ In memoriam \\ E-mail: sina.fietz@ipp.mpg.de
}

\begin{abstract}
.
Understanding the physics of transport during current ramps in tokamaks is essential in order to prepare adequate ramp-up and ramp-down scenarios for ITER, where constraints on the transformer flux and the internal inductance will be more stringent than in present-day devices. In this paper five transport models (CoppiTang, Neo-Alcator, Bohm/gyro-Bohm, critical gradient model and H98/2 scalingbased model) are used to reproduce the experimental data during the current ramps of ASDEX Upgrade with its metallic wall. The calculated temperature profiles are compared to the experimental temperature profiles under different ramp-up conditions. Our study reveals important differences between boronised and non boronised wall which are reproduced with variable success by the models. We also investigated rampup phases heated centrally by electron cyclotron heating which creates experimental conditions with peaked electron temperature profiles, which are very different from the usual ohmic heated cases. Our study reveals that the models react very differently to this additional heating.
\end{abstract}

PACS numbers: $52.55 \mathrm{Fa}, 52.50 . \mathrm{Gj}, 52.50 . \mathrm{Sw}$

Submitted to: Nuclear Fusion 


\section{Introduction}

The available transformer flux in ITER will be limited and the flux consumption during the current ramp must stay between certain limits such that enough flux remains available for the desired flat top length. In addition, the internal plasma inductance in ITER has to be low enough, in the range 0.7-1.0 at 15 MA due to control margins $[1,2]$. These limitations are set by the design of the poloidal field coil system to deliver enough flux for the flat top and to provide a stable plasma equilibrium [3]. It is therefore important to optimise the ramp-up phase of ITER and to be able to predict the behaviour of relevant plasma characteristics which in particular requires transport modelling. But, before being able to model ITER plasmas, the models have to be validated on present devices.

So far, mainly the properties of plasmas in the flat-top have been investigated and most of the transport models have been developed for these conditions. In contrast, the transport physics in the current ramp is little studied $[4,3]$. The main difference between the flat-top and the current ramp is the large variation of almost all plasma parameters and fast evolving profiles during the ramp. Here, the current is induced by the transformer coil at the plasma edge and diffuses into the plasma. Therefore, while the current density $\vec{j}(r)$ evolves from broad or hollow profiles to the typical peaked flattop profiles, Ohmic heating profiles with off-axis maxima can occur. The safety factor at the edge, $q_{95}$, is high at the beginning and decreases during the current ramp. The electron temperature evolves gradually from low values to its higher flat-top value while the plasma density is also generally increased through gas puffing. The confinement improves with rising plasma current. Due to the initial plasma-wall interaction the impurity level is high at the beginning of the ramp but decreases with time such that the effective charge values $\left(Z_{\text {eff }}\right)$ and the radiation losses may be high at the start but decrease as the ramp-up evolves.

The aim of this paper is to examine different transport models applied to a set of ohmic as well as ECRH-assisted current ramp-up phases in the ASDEX Upgrade tokamak. This study provides a wide set of different conditions for which the results of the five transport models are compared. Profiles from a variety of ramps have been analysed and compared to the simulation results, which provides a selective validation and evaluation of the models against ASDEX Upgrade data.

More precisely, five transport models for the heat conductivity are used to simulate in particular the electron temperature profiles in the ramp-up phases. Most of these models have already been used previously to simulate and predict the ITER ramp-up phase, [3],[5],[6],[7]. In previous modelling studies [3],[5],[6], [7], the internal inductance has been used to assess the quality of the modelling. We show below that this quantity does not provide a unique assessment of the modelling quality and that a direct comparison of the modelled temperature profiles with the experimental data is more appropriate. We only include the internal inductance in our sensitivity study because this is an important operational quantity, in particular for ITER. 
The paper is organised as follows. In section 2 the five models, the experimental setup and the assumptions used for the simulations are introduced. The influence of the initial conditions on the modelling results as well as sensitivity studies are presented in section 3. The modelling results are presented and discussed in section 5. Conclusions are drawn in the last section. 


\section{Transport simulation set-up}

In this section, the five electron heat diffusivity models and the assumptions used for the simulations are introduced. The experimental conditions of the ASDEX Upgrade current ramps are also illustrated.

\subsection{Heat conductivity models}

In order to model the $T_{\mathrm{e}}$ profiles during the current ramp-up phases of ASDEX Upgrade, five models for the electron heat diffusivity $\chi_{\mathrm{e}}$ are used in this work. Most of them have been developed to characterise L-modes during current flat-tops with no or little additional heating power. Three of these models have already been used to simulate the current ramps of different devices and applied to predict the ITER current ramp-up phase [3],[5], [6],[7]. In all discharges analysed the toroidal magnetic fields was constant and so the models are not influenced by changes in this. There is also no or only a weak dependence on $Z_{\text {eff }}$ of all the models. The models are described in the next paragraphs, where the units are: $n_{\mathrm{e}}$ in $\left[10^{19} \mathrm{~m}^{-3}\right], T_{\mathrm{e}}$ in $[\mathrm{keV}], P$ in $[M W], B$ in $[T]$ and all length in $[m]$.

\section{Neo-Alcator model}

The first model is the Neo-Alcator model, which was developed according to the INTOR scaling and partly based on the Neo-Alcator scaling given in [8], which is valid for low densities such as in the current ramp. Compared to this scaling, the Neo-Alcator model used here includes an additional dependence on the main ion mass number and a different dependence on the safety factor $\mathrm{q}$. This yields the electron heat conductivity:

$$
\chi_{\mathrm{e}}(r)=143 \epsilon \frac{1}{q(r)} \frac{1}{n_{\mathrm{e}}(r) R_{0} \sqrt{\frac{A_{\mathrm{i}}}{m_{\mathrm{p}}}}}
$$

The parameter $\epsilon$ is the inverse aspect ratio, $R_{0}$ the major radius, $n_{\mathrm{e}}$ the local electron density and $q$ the local safety factor. The ratio $A_{\mathrm{i}} / m_{\mathrm{p}}$ is the main ion mass number ( $m_{\mathrm{p}}$ is the proton mass) which is 2 for Deuterium plasmas such as at ASDEX Upgrade. This model has not been used to model current ramps in other studies, but in the following it is shown that the results of this simple model are comparable to those yielded by the usually used models.

\section{H98/2 scaling-based model}

The H98/2 scaling-based model also results from a confinement scaling for which the ITER IPB98(y,2) H-mode scaling is used [9]. This model has been also used to model L-modes or ohmic plasmas in previous publications using a correspondingly low enhancement factor $H_{98, \text { scal }}$, $[5,3]$. In the present work we assume that the confinement time, $\tau_{\mathrm{E}}$, in L-mode is half of the H-mode value. Following [5] and [3], we also assume 
that the electron heat diffusivity has a fixed parabolic shape in $\rho$, where $\rho$ is the usual normalised toroidal radius, according to the expression:

$$
\chi_{\mathrm{H} 98 / 2}=0.18\left[1+6 \rho^{2}+80 \rho^{20}\right]
$$

Of course the results of this model depend on the chosen shape for the heat diffusivity profile, but in this work we follow the assumptions made in [5] and [3].

The level of the diffusivity $\chi_{\mathrm{e}}$ is then adjusted by the time-dependent value $C_{\mathrm{H} 98 / 2}(t)$ such that $H_{98, \text { scal }}=0.5$ yielding:

$$
\chi_{\mathrm{e}}=C_{\mathrm{H} 98 / 2}(t) \cdot \chi_{\mathrm{H} 98 / 2}
$$

In the modelling this is achieved by a feed-back loop, described by equation 4 , which converges within less than $0.1 \mathrm{~s}$ after the start of the simulation.

$$
C_{\mathrm{H} 98 / 2}\left(t_{\mathrm{i}}\right)=C_{\mathrm{H} 98 / 2}\left(t_{\mathrm{i}-1}\right) \cdot\left(1-\left(H_{98, \text { scal }}-H_{98}\right)\right) \cdot \frac{H_{98}}{H_{98, s c a l}}
$$

The time step $t_{\mathrm{i}}-t_{\mathrm{i}-1}$ for the calculation of $C_{\mathrm{H} 98 / 2}(t)$ has been set to $1 \cdot 10^{-4} \mathrm{~s}$.

\section{Coppi-Tang model}

We also used the Coppi-Tang model, [10], whose heat diffusivity consists of three factors. One factor is the geometric mean of two contributions to the total electron heat transport: that caused by the electron-driven turbulence, $\chi_{\mathrm{TEM}}$, and that due to the ion-driven turbulence, $\chi_{\eta i}$. A second factor, the profile factor $F(\Phi)$, which has an exponential radial dependence, provides an increase towards the plasma edge. The variable $\Phi$ is the toroidal flux, used as the radial coordinate in this model. Finally, the third factor $f_{m}$ models the effect of sawteeth. It is equal to 1 outside the $q=1$ surface and 4 inside it which then creates very flat $T_{e}$ profiles, as will be shown later. The model is described by the following equations:

$$
\begin{aligned}
& \chi_{\mathrm{e}}=\frac{1}{|\nabla \Phi|^{2}} \sqrt{\left(\chi_{\mathrm{TEM}}\right)^{2}+\left(\chi_{\eta \mathrm{i}}\right)^{2}} \cdot F(\Phi) \cdot f_{\mathrm{m}} \\
& \text { with } \\
& \chi_{\mathrm{TEM}}=a_{122}\left(1.25 \cdot 10^{20}\right) \frac{a}{n_{\mathrm{e} 0}}\left(R_{0} B_{0}\right)^{0.3} Z_{\mathrm{eff}}^{0.2} \cdot\left(1+\frac{1}{4} \alpha_{\mathrm{n}}\right) R_{0}^{-2.2} q_{95}^{-1.6} \\
& \chi_{\eta \mathrm{i}}=a_{121}\left(7.5 \cdot 10^{8}\right)\left[\frac{P_{\mathrm{tot}}}{n_{\mathrm{e} 0}}\right]^{0.6}\left(R_{0} B_{0} q_{95}\right)^{-0.8} a^{-0.2} \\
& F(\Phi)=8 \pi^{2} \frac{p_{\text {tot }}(\Phi)}{P_{\text {tot }}} \frac{n_{\mathrm{e} 0}}{n_{\mathrm{e}}(\Phi)} \frac{R_{0} \Phi_{\mathrm{b}}}{\partial V / \partial \Phi} \cdot e^{\left[\frac{2}{3} \alpha_{\mathrm{q}} \frac{\Phi}{\Phi_{\mathrm{b}}}\right]}
\end{aligned}
$$

The plasma minor radius $a$ is defined as $\sqrt{V_{\mathrm{b}} / 2 \pi R_{0}}$ ( $V_{\mathrm{b}}$ is the total volume), $n_{\mathrm{e} 0}$ is the central electron density, $B_{0}$ the toroidal magnetic field, $\Phi_{b}$ the toroidal flux at the 
plasma boundary. The parameter $\alpha_{\mathrm{q}}$ is defined as $q_{95}+0.5$. The values of the constants $a_{122}, a_{121}, \alpha_{\mathrm{n}}$ are 0.40, 0.08 and 0.5, respectively, as set in [10]. Note in this model the strong dependence on parameters which vary during the ramp, such as $n_{e 0}, q_{95}, P_{\text {tot }}$ and its radial distribution $p_{\text {tot }}(\Phi)$, while the dependence on $Z_{\text {eff }}$ is weak. The exponential part of the profile factor is dominant for ohmic discharges, while $P(\Phi)$ becomes important for cases with ECRH due to its very localised deposition profile, as will be shown in section 5 .

\section{Bohm/gyro-Bohm}

The mixed Bohm/gyro-Bohm model $(\mathrm{B} / \mathrm{gB})$ is largely used for JET plasmas, $[11,12,13]$. The version used in this paper is mainly based on the original L-mode version introduced in [14]. The electron heat diffusivity is the sum of two contributions with different weights, one with a Bohm dependence, labelled $\chi_{B}$, and one with a gyroBohm dependence, $\chi_{g B}$ :

$$
\begin{aligned}
& \chi_{\mathrm{e}}=\chi_{\mathrm{B}}+\chi_{\mathrm{gB}} \\
& \text { with } \\
& \chi_{\mathrm{B}}=\alpha_{\mathrm{B}} \frac{c T_{\mathrm{e}}}{e B_{0}} q^{2} \frac{a}{\left|L_{\mathrm{p}_{\mathrm{e}}}\right|} \\
& \chi_{\mathrm{gB}}=\alpha_{\mathrm{gB}} \frac{c T_{\mathrm{e}}}{e B_{0}} \frac{a}{\left|L_{\mathrm{T}_{\mathrm{e}}}\right|} \rho_{s}
\end{aligned}
$$

The variables of the form $L_{\mathrm{y}}$ are the usual gradient length of the radial profile of quantity $y: L_{\mathrm{y}}=-y / \nabla y$. The heat diffusivity $\chi_{\mathrm{B}}$ is of the Bohm-type, hence depending on $T_{\mathrm{e}}, q^{2}$ and $L_{\mathrm{p}_{\mathrm{e}}}$, the gradient length of the electron pressure, and assuming that turbulence scales with the machine size. The term $\chi_{\mathrm{gB}}$ is of the gyro-Bohm-type which involves a stronger dependence on $T_{\mathrm{e}}$, namely $T_{\mathrm{e}}^{3 / 2}$, due a dependence on the gyro radius $\rho_{s}$, which is defined as $\sqrt{m_{\mathrm{i}} T_{\mathrm{e}}} / e B_{0}\left(m_{\mathrm{i}}\right.$ is the main ion mass), and a dependence on $L_{\mathrm{T}_{\mathrm{e}}}$, the temperature gradient length. The parameter $Z_{\mathrm{i}}$ is the charge of the main ions, which is one in Deuterium plasmas. The $\chi_{\mathrm{gB}}$ contribution assumes turbulence scale of the order of the gyro-radius. The numerical coefficients $\alpha_{\mathrm{B}}$ and $\alpha_{\mathrm{gB}}$ are $2.5 \cdot 10^{-4}$ and $3.5 \cdot 10^{-2}$, [11].

\section{Critical Gradient Model}

The fifth model is the critical gradient model (CGM), which has been widely used in ASDEX Upgrade [15]. This model is based on the assumption that electron heat transport is driven by the TEM instability $[16,17]$. It is of the gyro-Bohm type and one assumes that the electron heat diffusivity increases above a threshold in $R / L_{T e}$, labelled $R / L_{T e, c r i t}$, whose existence, which is predicted by theory [18][19], has been evidenced experimentally [20]. Below the threshold the transport is very low. It also includes a $q^{3 / 2}$ dependence required to reproduce the increase of heat transport towards the plasma edge, as generally observed experimentally. The electron heat diffusivity is then written as: 


$$
\begin{aligned}
& \chi_{\mathrm{e}}=\chi_{0} q^{3 / 2} \frac{T_{\mathrm{e}} \rho_{\mathrm{s}}}{e B_{0} R_{0}} \\
& +\chi_{\mathrm{s}} q^{3 / 2} \frac{T_{\mathrm{e}} \rho_{\mathrm{s}}}{e B_{0} R_{0}}\left(\frac{R_{0}}{L_{\mathrm{Te}}}-\frac{R_{0}}{L_{\mathrm{Te}, \text { crit }}}\right) H\left(\frac{R_{0}}{L_{\mathrm{Te}}}-\frac{R_{0}}{L_{\mathrm{Te}, \text { crit }}}\right)
\end{aligned}
$$

The gyro-radius $\rho_{\mathrm{s}}$ is defined above. The function $H$ is the Heaviside function which is zero if $\frac{R_{0}}{L_{\mathrm{Te}}}$ is lower than $\frac{R_{0}}{L_{\mathrm{Te}, \text { crit }}}$ and hence $\chi_{\mathrm{e}}$ is equal to the first term in equation 11. Otherwise $\mathrm{H}$ is equal to one and $\chi_{\mathrm{e}}$ strongly increases by the addition of the second term in equation 11. For the following investigations, the three free parameters, i.e. the background transport $\chi_{0}$, the stiffness $\chi_{\mathrm{s}}$ and the threshold $R_{0} / L_{\mathrm{Te}, \text { crit }}$ are adjusted to ASDEX Upgrade current ramps. The background transport $\chi_{0}$ and the stiffness $\chi_{\mathrm{s}}$ are assumed to be constant and set to 0.5 and 0.3 respectively. The threshold $R_{0} / L_{\mathrm{Te} \text {,crit }}$ increases linearly from 1.0 at the beginning to 3.0 at the end of the current ramp in order to be consistent with the value in the flat top $[17,16]$.

\subsection{Transport modelling}

To model the current ramp-up phases, we use the transport code ASTRA, [21], in which for our study the time-dependent heat transport and the current diffusion equations are solved. In ASTRA the modelling is done with a prescribed boundary condition and a 3-momentum approach for the equilibrium reconstruction [22]. The experimental values for the elongation, the triangularity, the minor and the major radius are delivered by the equilibrium code CLISTE [23] and plugged into ASTRA. The equilibrium reconstruction in ASTRA is simplified so no up-down asymmetries are taken into account.

For the simulation the electrical conductivity is calculated neoclassically, [24], and the electron heat conductivity is provided by the five models described above. In our study we mainly compare the $T_{e}$ profiles yielded by the models to the experimental ones. In contrast to the steady-state studies in the flat-top, the choice of the initial $T_{\mathrm{e}}$ and the current density profiles may be important, due to the profile evolution during the current ramp. The relaxation time from the initial profiles to those fully determined by the models can influence the results. This issue is investigated in section 3.

The electron heating power taken into account in the simulations includes the ohmic heating power, the collisional electron-ion heat exchange and a term for ECRH, if applied. The radiation losses are taken into account. In the discharges presented here, no direct ion heating was applied and the ion heat flux is purely due to the electron-ion collisional exchange. The electron density $\left(n_{\mathrm{e}}\right)$, the radiation power density $\left(p_{\text {rad }}\right)$ and the $Z_{\text {eff }}$ profiles are based on measured data, [25], [26] and [27] respectively. No ion temperature measurements are available in these ramp-up studies and the $T_{i}$ profile is calculated using the neoclassical ion diffusivity, [28, 29]. This assumption has been validated by comparisons between the simulated and experimental stored energy $W_{\text {mhd }}$. Furthermore, for two models, Coppi-Tang, [10], and Bohm/gyro-Bohm, [11], we also compared the results yielded by the corresponding ion heat conductivity. The different 
choices for $T_{\mathrm{i}}$ yield rather comparable results for $W_{\text {mhd }}$ and had only a weak impact on the results of the models for $T_{\mathrm{e}}$. Therefore we assumed a neoclassical ion heat transport for the whole study.

The electron heat flux is related to $\chi_{\mathrm{e}}$ and $\nabla T_{\mathrm{e}}$, whereas $\chi_{\mathrm{e}}$ itself can depend on $T_{\mathrm{e}}$, $\nabla T_{\mathrm{e}} / T_{\mathrm{e}}, \vec{j}(r)$ or $P_{\text {heat }}$ depending on the models, as described above. Therefore the coupling between the transport equation system depends on the transport model.

As indicated above, for the validation of the $\chi_{\mathrm{e}}$ models, we compare the modelled and experimental $T_{\text {e }}$ profiles which are provided by the electron cyclotron emission diagnostic (ECE). The experimental $T_{\mathrm{e}}$ profiles measured with the Thomson scattering exhibit a very large data scatter at the beginning of the ramp due to the quite low density. In the later phase of the current ramp-up, they are consistent with the ECE data. There the ECE and Thomson scattering $T_{e}$ profiles have been cross-checked where possible but only the ECE data are shown in the next sections. It should be emphasised here that in our discharges neither $T_{e}$ data from ECE nor from Thomson scattering, are available inside $\rho_{\text {tor }} \approx 0.2-0.3$.

The internal inductance deduced from the equilibrium code and that yielded by modelling are compared in later sections. Both are defined as $l_{\mathrm{i}}(3)$, see e.g. [30] [31]:

$$
l_{\mathrm{i}}(3)=\frac{2}{\left(\mu_{0} I_{\mathrm{p}}\right)^{2} R_{\text {geo }}} \int B_{\text {pol }}^{2} d V
$$

with $R_{\text {geo }}$ being the radius of the geometric axis of the plasma, $B_{\text {pol }}$ the poloidal magnetic field, $\mathrm{V}$ the plasma volume and $I_{\mathrm{p}}$ the plasma current. At ASDEX Upgrade, the experimental equilibrium and the corresponding internal inductance are provided by the CLISTE code.

\subsection{Experimental conditions}

ASDEX Upgrade is a medium size tokamak with major radius $R_{0}$ of $1.65 \mathrm{~m}$, minor radius $a$ of $0.5 \mathrm{~m}$ and a maximal pulse duration of about $10 \mathrm{~s}$. Usually the current ramp-up phase takes about 1 s to reach $0.8 \mathrm{MA}$, which is a typical value for the flat-top current and used in the discharges presented here. The magnetic field is constant over time at about 2.0-2.5 $\mathrm{T}$ in the analysed discharges. The standard current ramps are ohmically heated and additional heating power is only used for particular purposes. In this paper we analysed ohmically and ECRH heated ramps. The inner wall was fully coated with tungsten [32], boronised or not, and the two cases lead to very different ramp-up conditions as will be shown in the next section. ASDEX Upgrade has been run for several experimental campaigns with non-boronised tungsten wall such that the plasma facing component surfaces were tungsten coated [33], [34]. The non-boronised example presented in this work is taken from this period.

In figure 1 plasma parameters of an ohmic current ramp-up for a non-boronised discharge are shown. This discharge is in the L-mode during the whole current ramp, which is the usual case. The plasma current is increased almost linearly with the usual ramp rate. 


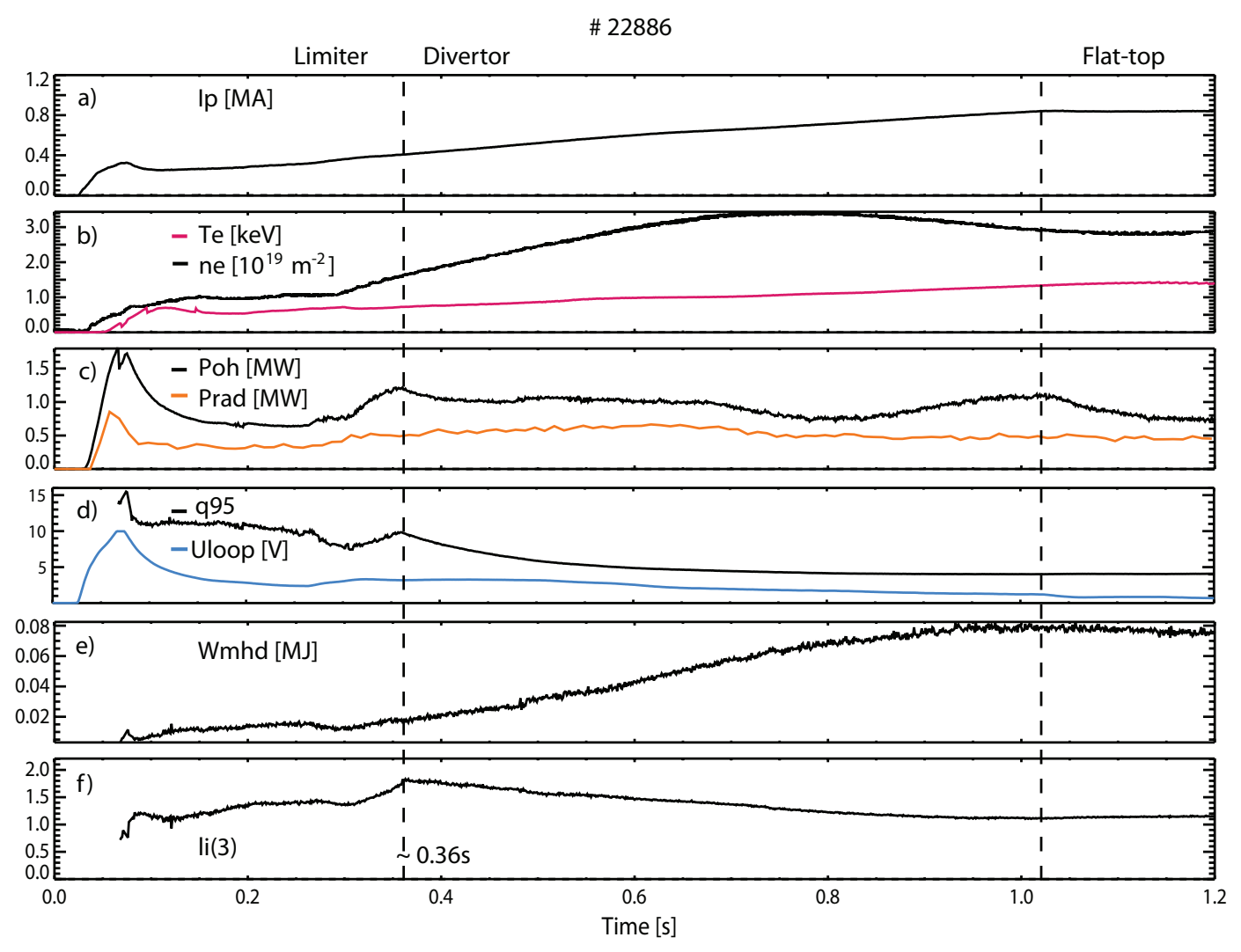

Figure 1. Plasma parameters of an ohmic ASDEX Upgrade current ramp-up phase: a) plasma current, b) electron temperature measured with ECE inside $\rho_{\text {pol }}=0.5$ and line integrated density from a central interferometer chord, c) ohmic heating power and radiation power, d) loop voltage and safety factor at the edge, e) stored plasma energy and f) internal inductance. The transition from a limiter to the X-point plasma and the beginning of the flat-top are indicated by vertical lines.

The density is increased by gas puffing and its value is kept rather low to save ohmic flux and also avoid the density limit. With rising plasma current the stored energy, electron temperature and confinement increase. The loop voltage and $q_{95}$ decrease strongly just after the break-down $(\mathrm{t} i, 0.1 \mathrm{~s})$ and later evolve gradually to reach their flat-top values. In figure $1 \mathrm{c}$ ) the ohmic heating power and the typically large amount of radiation power loss due to the non-boronised wall are shown. The experimental internal inductance, shown in panel f, reaches its highest value before the transition into the divertor configuration. This highest value can reach about 2 in the non-boronised cases, but generally remains below 1.5 for boronised discharges.

The plasma shape evolves strongly during the current ramp, which is illustrated in figure 2. At the beginning the plasma is circular in limiter configuration. During the ramp-up phase the elongation is increased and the plasma evolves into a diverted plasma. For the discharge presented in figure 1 and 2 the X-point formation occurs at about $0.36 \mathrm{~s}$.

In the current ramp the demands on transport models are high. The plasma shape is evolving from a circular to an elongated X-point plasma and all plasma parameters are 

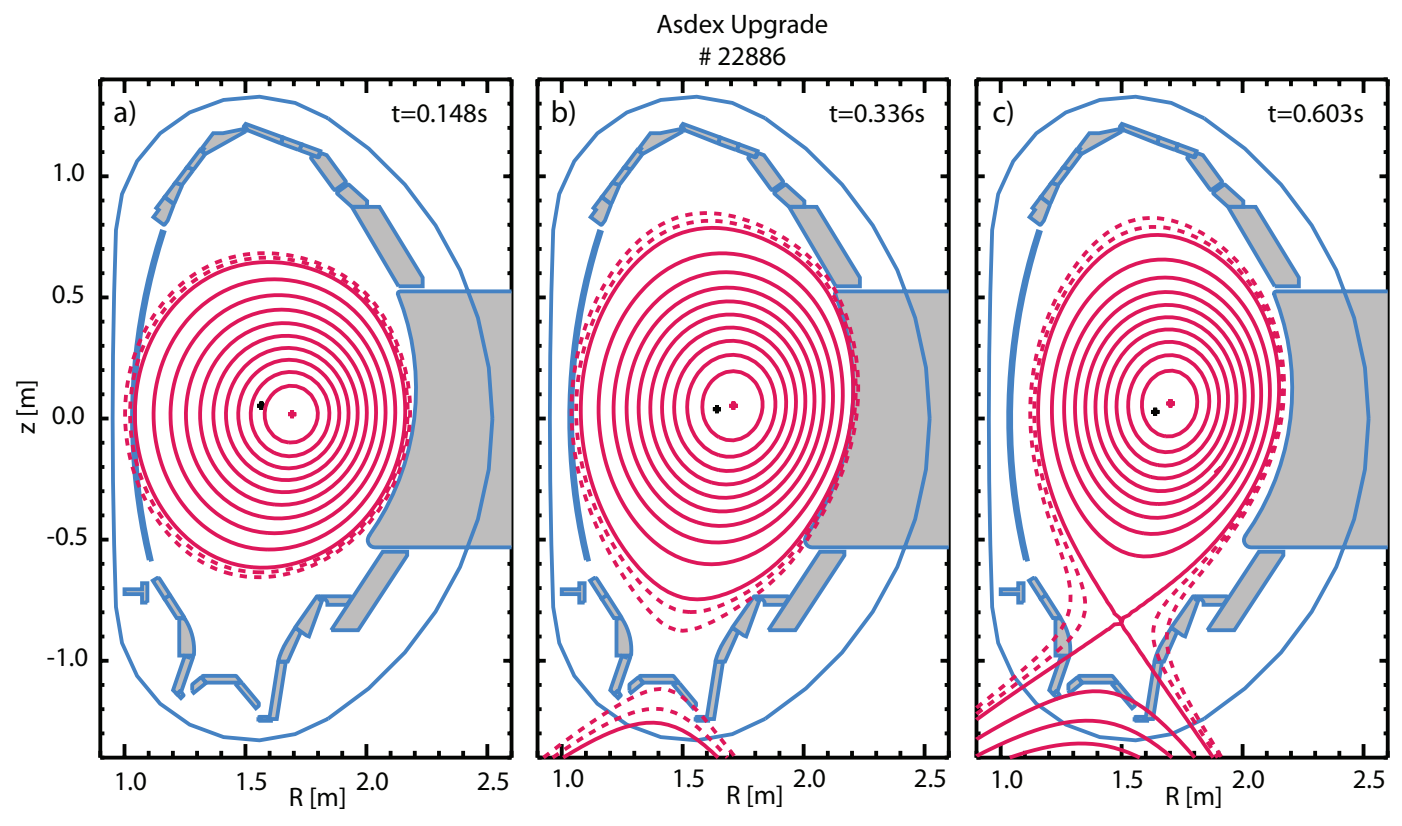

Figure 2. Illustration of the plasma shape evolution during a current ramp at a) 0.15 s round inner limiter plasma, b) 0.34 s elongated outer limiter plasma and c) $0.60 \mathrm{~s}$ $\mathrm{X}$-point plasma

continuously changing and cover a wide range of values. The transport models must be able to deal with fast evolving plasma conditions and hence, in particular, the modelling results may not be influenced by the initial conditions. 


\section{Sensitivity studies on the internal inductance}

The internal inductance is an important parameter for plasma stability and shaping. Therefore, in most of the experimental and modelling studies for ITER predictions and scenarios, the internal inductance is extensively discussed, see e.g. [3, 1].

This section is not about the heat transport models but more about the current diffusion and deals with the important question if the internal inductance is a sufficient parameter for the validation of transport models.

In previous works the internal inductance is often used to validate transport models and we show below that this does not always yield an unambiguous answer.

\subsection{Comparison of experimental and simulated plasma inductance}

First of all, we compare the CLISTE internal inductance determined from magnetic measurements with $l_{\mathrm{i}}(3)$ calculated by ASTRA. In this comparison, the $T_{e}$ profiles are not modelled but the experimental time-evolving $T_{\mathrm{e}}$ profiles are used and the current diffusion is calculated accordingly with the neoclassical conductivity. In addition, the influence of the initial $q$ profile is also investigated in which three different initial $q$ profiles are used, see figure 3 a). These $q$ profiles are inverted, hollow and flat and taken from CLISTE at $0.12 \mathrm{~s}, 0.15 \mathrm{~s}$ and $0.2 \mathrm{~s}$ respectively. The simulations are started in each case at one of these points with the corresponding profile. The three time evolutions of $l_{\mathrm{i}}(3)$ from ASTRA are compare to those from CLISTE in figure $3 \mathrm{~b}$ ).
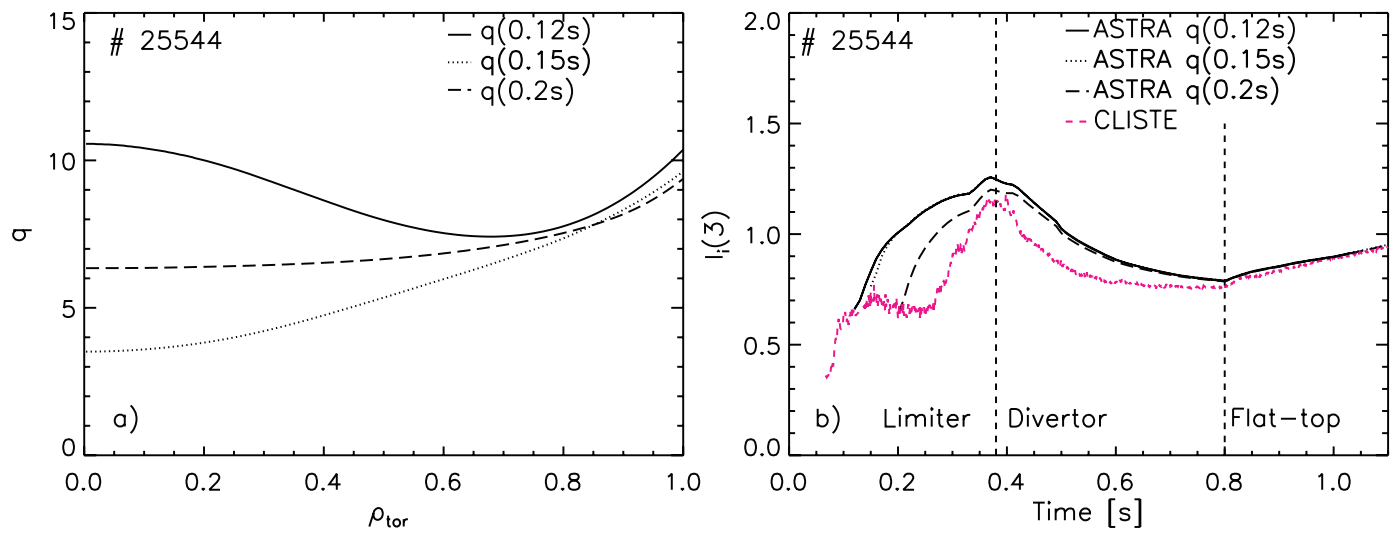

Figure 3. a) Three initial $q$-profiles calculated with CLISTE at three different time-points; b) Comparison of internal inductance from CLISTE and calculated with ASTRA. For ASTRA the evolution is analysed based on the three different initial q-profiles illustrated in a).

The CLISTE value of $l_{\mathrm{i}}(3)$ first increases, levels off from $0.15 \mathrm{~s}$ to $0.25 \mathrm{~s}$, after which it increases again to reach a maximum at the time of the transition to the divertor configuration. In the divertor configuration $T_{e}$ increases strongly with time, in particular in the edge region, which, together with the $\mathrm{OH}$ induced current, causes the decrease of $l_{\mathrm{i}}(3)$ until the end of the current ramp at about $0.8 \mathrm{~s}$. 
The overall trend of the CLISTE time evolution is well reproduced by the ASTRA simulation, in particular the increase before the transition to the divertor configuration and its decreases after it. Only at the very beginning of the current ramp the ASTRA simulation deviates from the CLISTE result. There are two reasons for this deviation: Shortly after the breakdown MHD activity occurs, which possibly influences the q profile evolution. Furthermore the $T_{\mathrm{e}}$ measurements at the edge with ECE are not reliable at the beginning of the current ramp due to its low values. This results in additional uncertainties of the $l_{\mathrm{i}}(3)$ of ASTRA before $0.4 \mathrm{~s}$. After the transition to the X-point plasma these deviations decrease due to a reliable CLISTE equilibrium reconstruction and good experimental $T_{\text {e }}$ profiles used for the ASTRA simulation.

It can also be seen that the time evolution is almost not affected by the initial $q$ profiles. Quantitatively, $l_{\mathrm{i}}(3)$ from ASTRA exhibits a trend to be somewhat larger than the CLISTE result. This difference, which is observed in all our simulations is attributed to the simplified ASTRA equilibrium, [21, 22], which, includes the elongation, the triangularity and the Shafranov shift of the plasma, but no up-down asymmetry and X-point.

\subsection{Sensitivity of $l_{\mathrm{i}}(3)$ on the $T_{\mathrm{e}}$ profile}

The internal inductance is a normalised volume-averaged quantity. It strongly depends on the radial distribution of the current density which is directly influenced by the temperature profile. In this section the influence of different $T_{\mathrm{e}}$ profiles on the time evolution of $l_{\mathrm{i}}(3)$ is analysed. It is shown that considering the internal inductance only, and not the $T_{\mathrm{e}}$ profiles, can lead to a misinterpretation of the model performance.

For this investigation four different $T_{\mathrm{e}}$ profiles around the experimental one have been defined. As indicated above and shown in figure 4 a) no ECE data are available for $\rho_{\text {tor }} ; 0.3$, leaving quite some freedom for the shape of the profiles. In this area two assumptions were made, a peaked and a hollow $T_{\mathrm{e}}$ profile, respectively green and blue in figure $4 \mathrm{a}$ ). For $\rho_{\text {tor }}$ \& 0.3 we made assumptions which are largely above the nominal ECE experimental uncertainties of $7 \%$ provided by two further profiles. One of them, orange in Fig. 4, is based on the hollow profile but with a much lower temperature in the edge region, which shows how the effect of the low temperature in the centre on $l_{\mathrm{i}}(3)$ can be compensated by a lower edge temperature. Finally, the fourth profile (red) has a shape similar to the hollow profile but with higher $T_{\mathrm{e}}$ over the whole radius. This is larger than the nominal uncertainties of ECE, but remains lower than the deviations of the modelled $T_{\mathrm{e}}$ profiles, as we will see later. During the time evolution of the calculations, these $T_{\mathrm{e}}$ profiles are proportional to the experimental reference profile keeping their respective shapes. In figure 4 a) only one snap shot during the current ramp is shown. In figure $4 \mathrm{~b}$ ) the profiles of the current density $j$ corresponding to the different $T_{\mathrm{e}}$ profiles at $0.3 \mathrm{~s}$ are shown.

The peaked $T_{e}$ profile induces a clearly more peaked current density, whereas the low edge temperature (orange) strongly reduces the current density in the edge. 


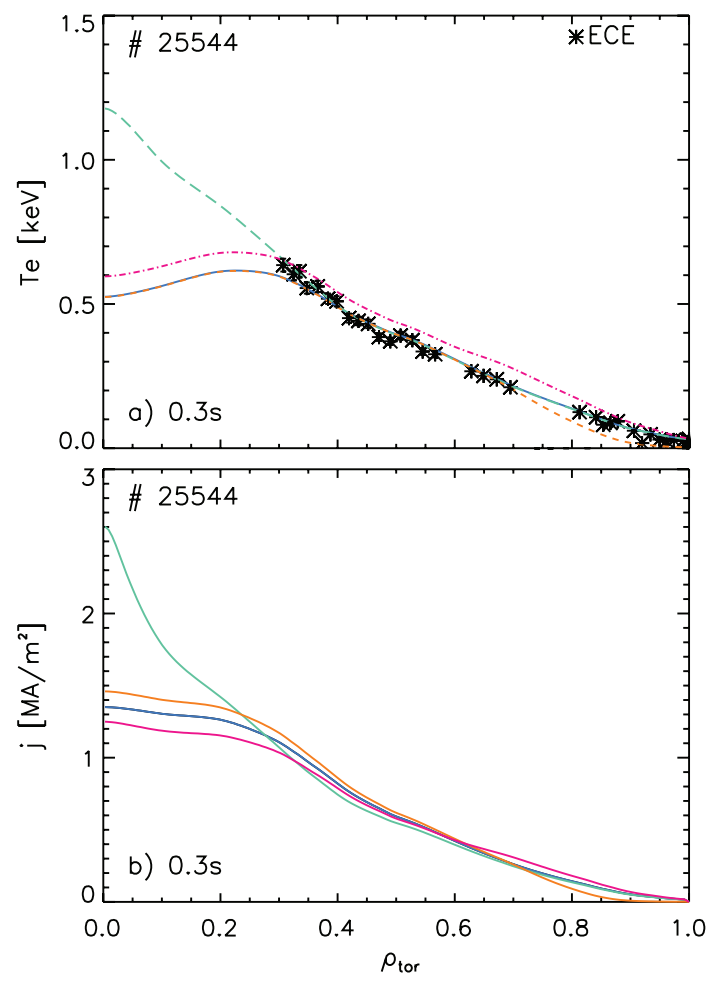

Figure 4. Sensitivity analysis of the influence of different $T_{\mathrm{e}}$ profiles on the internal inductance. a) Four variations of electron temperature profiles exemplary shown at $0.3 \mathrm{~s}$. The different line styles are used to better distinguish the four profiles. In the following pictures the associated curves are only colour-coded. b) Corresponding calculated parallel current density profiles at $0.3 \mathrm{~s}$.

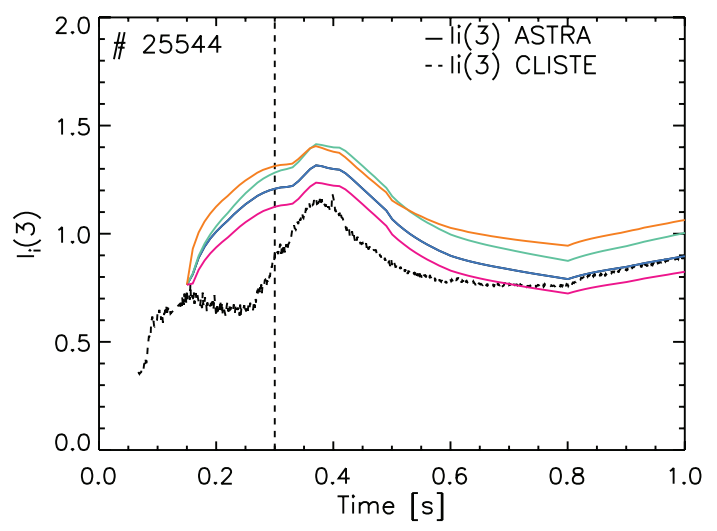

Figure 5. Time evolution of the internal inductance $l_{\mathrm{i}}(3)$ based on the different $T_{\mathrm{e}}$ profiles (figure 4$)$ and $l_{\mathrm{i}}(3)$ from CLISTE. The dashed vertical line indicates the time point of the exemplary shown $T_{\mathrm{e}^{-}}$and $j$ profiles. 
The internal inductance resulting from these profiles are shown in figure 5, together with the CLISTE internal inductance. A higher $T_{\mathrm{e}}$ in the core results in a higher $l_{\mathrm{i}}(3)$, due to the resulting lower resistivity in the central region. Indeed, this leads to a more peaked current density profile, as can be seen in the $j$ profiles (compare blue and green). In contrast, the internal inductance increases with decreasing edge temperature. This leads again to a diffusion of the current towards the centre of the plasma and a more peaked $j$ profile, compare blue and orange. The internal inductance is strongly weighted by the edge of the plasma, due to the volume effect and variations at the edge dominate over deviations in the central region. This is well illustrated by the orange and green $l_{\mathrm{i}}(3)$ curves which are comparable despite very different $T_{e}$ profiles. A change of the whole $T_{\mathrm{e}}$ profile has an impact on $l_{\mathrm{i}}(3)$ which is dominated by the deviations near the edge, as can be seen on the basis of the red profile which results in a less peaked $j$ profile compared to the blue profile. This leads to a lower $l_{\mathrm{i}}(3)$ due to the increased $T_{\mathrm{e}}$ towards the edge despite a higher core $T_{\mathrm{e}}$. The variations of the $T_{\mathrm{e}}$ profiles investigated in this section remain roughly within the uncertainties of the experimental $T_{\mathrm{e}}$ profiles but the influence on $l_{\mathrm{i}}(3)$ is significant. As shown in the next section, the variations of the $T_{\mathrm{e}}$ profiles calculated with the $\chi_{e}$ models compared to the measured data largely exceed the variation used here. In the same way the impact on $l_{\mathrm{i}}(3)$ also increases. Thus, evaluating the quality of the $\chi_{\mathrm{e}}$ models on $l_{\mathrm{i}}(3)$ can be highly misleading. In the following, the examination and the validation of the $\chi_{\mathrm{e}}$ models will only be based on the evolution of the predicted temperature profiles. The influence of the models on $l_{\mathrm{i}}(3)$ is not discussed.

\subsection{Sensitivity of $l_{\mathrm{i}}(3)$ on the $Z_{\text {eff }}$ profile}

In addition to the $T_{\mathrm{e}}$ profiles, the effective ion charge profiles $\left(Z_{\text {eff }}\right)$ are important for the current density evolution and internal inductance. At ASDEX Upgrade the $Z_{\text {eff }}$ profiles are provided by the integrated data analysis (IDA), which includes the data from the bremsstrahlung measurements, [27]. The uncertainties on $Z_{\text {eff }}$ are about $20 \%$ in the core and larger at the edge. This study deals with the impact of the experimental uncertainties on the current profile development. As the experimental uncertainties are independent of other quantities, dilution and radiation power are kept constant in this study.

To investigate the influence of these large errors of $Z_{\text {eff }}$ on the current profile, the $Z_{\text {eff }}$ profile was varied arbitrarily around the experimental value, but kept constant over time for this qualitative study. The different $Z_{\text {eff }}$ profiles are shown in figure 6 a) together with the reference experimental $Z_{\text {eff }}$ profile taken at $0.31 \mathrm{~s}$. To yield clear effects, the variations were chosen to be significantly larger than the nominal experimental uncertainties. They are also larger at the plasma edge than in the core to reflect the larger uncertainties there.

The calculated current density profiles at $0.3 \mathrm{~s}$ corresponding to the $Z_{\text {eff }}$ profile are illustrated in figure $6 \mathrm{~b}$ ). The time evolution of $l_{\mathrm{i}}(3)$ based on these $Z_{\text {eff-profiles }}$ is 

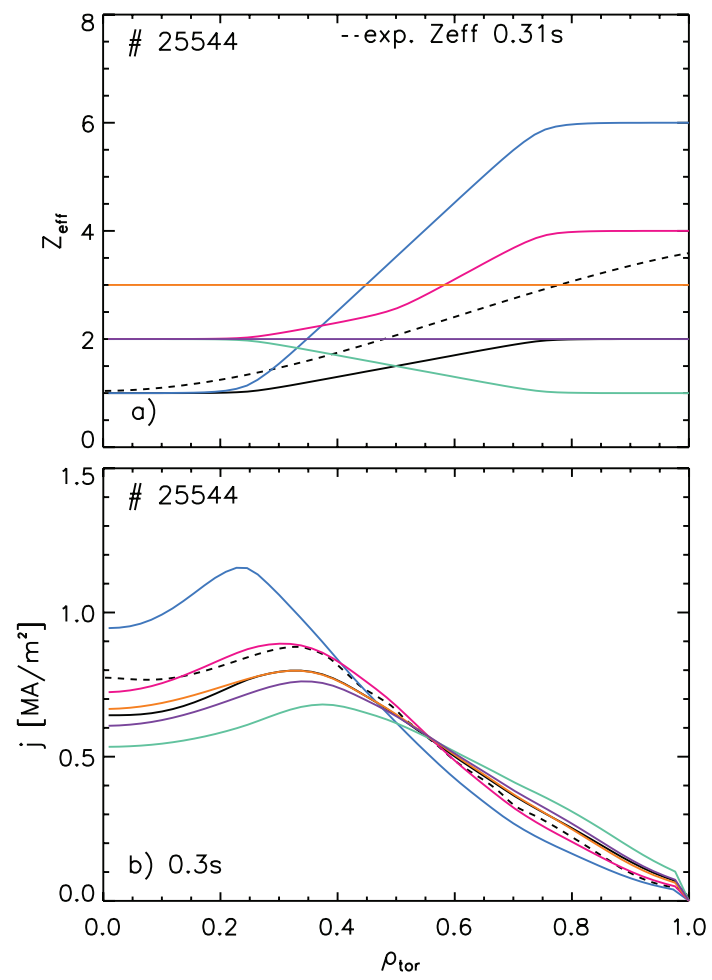

Figure 6. Analysis of the influence of different $Z_{\text {eff }}$ profiles (a) on current density profile (b). The colour code is the same for all three plots.

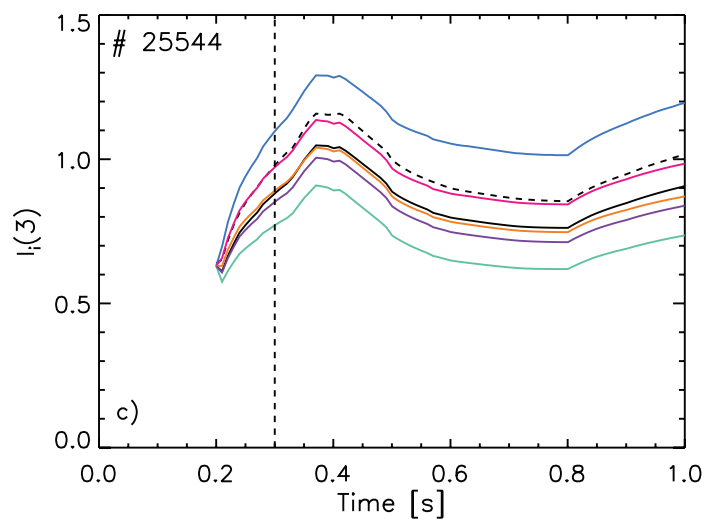

Figure 7. Analysis of the influence of different $Z_{\text {eff }}$ profiles (figure 6) on the internal inductance. The vertical dashed line indicates the time point of the exemplary $Z_{\text {eff }}$ and $j$ profiles.

shown in figure 7 . The different $l_{\mathrm{i}}(3)$ time traces evolve parallel to each other because the $Z_{\text {eff }}$ profiles are time-independent. The overall result is that the shape of the $Z_{\text {eff }}$ profile has a strong influence on both $l_{\mathrm{i}}(3)$ and current density while scaling the whole profile up and down has almost no impact. This latter effect is illustrated by the violet and orange curves corresponding to flat $Z_{\text {eff }}$ profiles. As expected, an increase of $Z_{\text {eff }}$ at the edge (blue case) leads to a peaked current profile and very high $l_{\mathrm{i}}(3)$, while a reduction of the edge $Z_{\text {eff }}$ (red to green through violet cases) has the opposite effect. 
The very large differences between the extreme cases (green and blue) are probably out of the experimental variations, in particular the green low edge case. The other profiles are realistic and lead to a maximum variation of $l_{\mathrm{i}}(3)$ of about 0.15 which is comparable to the overall variation of $l_{\mathrm{i}}(3)$ over time. Therefore within realistic experimental uncertainties, the effect of $Z_{\text {eff }}$ is not negligible but it does not dominate the behaviour of $l_{\mathrm{i}}(3)$.

\section{Influence of the initial $T_{e}$ and $j$ profiles on the modelling results}

The simulations are started after the plasma breakdown at about $0.1 \mathrm{~s}-0.2 \mathrm{~s}$ when the discharge is still a circular limiter plasma. At this time point, the initial profiles for the safety factor and $T_{\mathrm{e}}$ are provided. The influence of these initial profiles on the modelling is investigated. Their influence cannot be predicted in advance due to the nonlinear transport equation system and the different dependencies of the models. Furthermore, their impact is different for each model.

\subsection{Initial $T_{\mathrm{e}}$ profile}

In this paragraph, we investigate the influence of two different initial $T_{\text {e }}$ profiles on the calculated $T_{\mathrm{e}}$ profiles resulting from the different models. We use two experimental profiles, one is peaked (with flat central region) and the other hollow, as shown in figure 8. In both cases a flat initial q-profile is used.

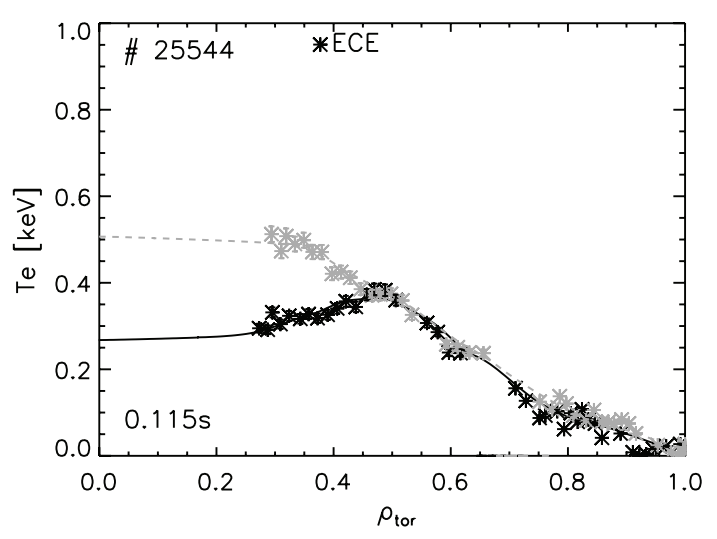

Figure 8. The two initial $T_{\mathrm{e}}$ profiles used to examine their influence on the modelling. The hollow profile is in black and the peaked profile (with flat central region) in grey.

The $T_{\mathrm{e}}$ profiles calculated with the five models, starting with these two different initial profiles, are illustrated in figure 9 for three time points. The first time point, panel a), is taken at $0.25 \mathrm{~s}$ which is only $0.135 \mathrm{~s}$ after the start of the simulation. Even shortly after the start of the simulation, the influence of the initial profile on the predicted $T_{\mathrm{e}}$ profiles is small for all five models. This is due to the short energy confinement time at the beginning of the current ramp. The largest difference caused by the different initial $T_{\mathrm{e}}$ profiles appears for the Coppi-Tang model, but it remains much smaller than the 
overall deviation from the experimental data and differences between the models. For later times the differences are smaller. Therefore, the influence of the initial $T_{\mathrm{e}}$ profile can be neglected for all analysed $\chi_{\mathrm{e}}$ models.
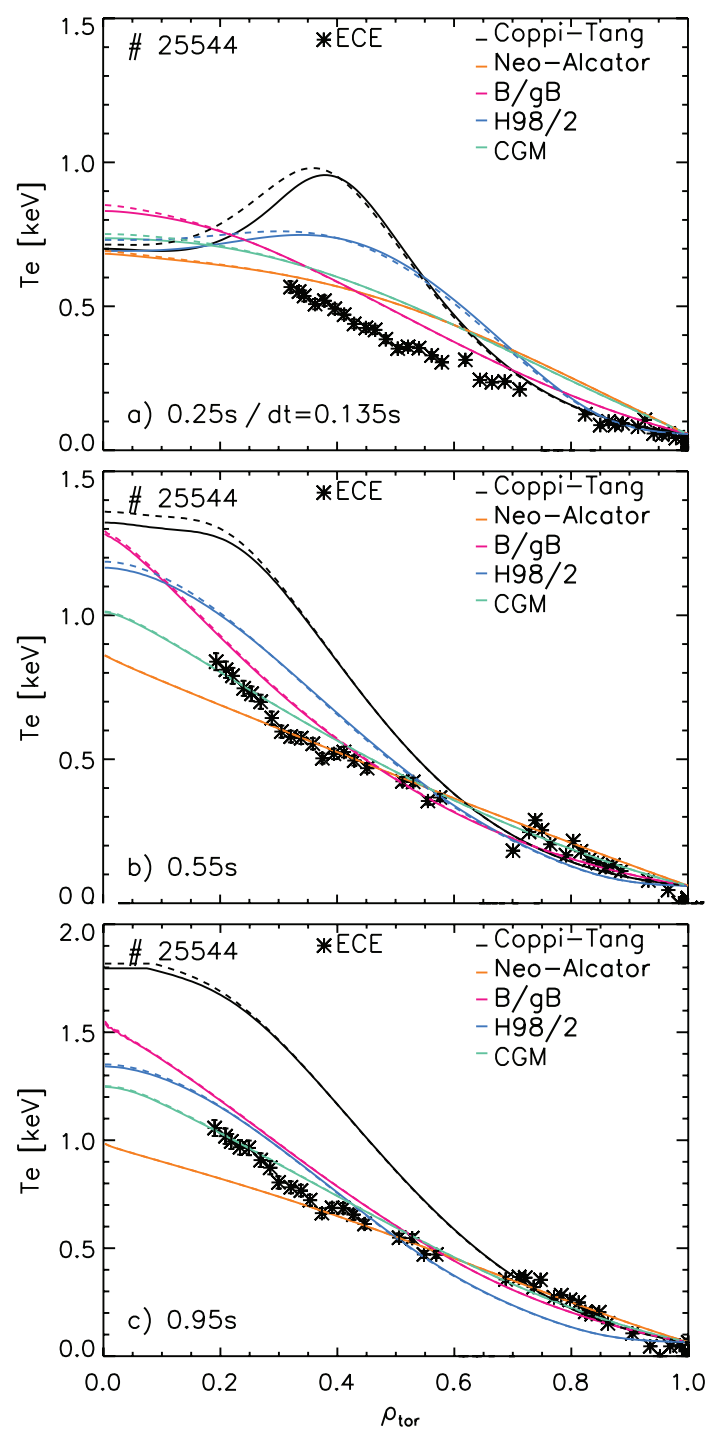

Figure 9. $T_{\mathrm{e}}$ profiles calculated by the models based on different initial $T_{\mathrm{e}}$ profiles according to figure 8: a) at the beginning of the ramp, b) in the middle and c) at the end. The solid line profiles are calculated using the hollow initial $T_{\mathrm{e}}$ profile (black) and the dashed profiles using the peaked initial $T_{\mathrm{e}}$ profile (grey).

\subsection{Initial $j$ profile}

The $T_{\text {e }}$ profiles yielded by the models can be influenced by the initial $j$ profile through their dependence on $q$ and Ohmic heating power. The influence of the $j$ profile can be particularly important in the cases without auxiliary heating. The initial current density distribution is taken from the experimental equilibrium yielded by CLISTE. In the following we discuss the $j$ profile by its corresponding $q$ profile which is a more 
familiar quantity. These profiles have very large uncertainties due to the absence of direct measurements and it is essential to assess how they may affect the modelling results. The impact of the initial $j$ profile on the modelled $T_{\mathrm{e}}$ profiles is different for each model. It depends on the dependence of $\chi_{e}$ on the $q$ profile directly and the ohmic heating power, as well as on $T_{\mathrm{e}}$ and $\nabla T_{\mathrm{e}}$. For this purpose, we analysed the influence of a flat, a hollow and a reversed initial $q$ profile, on the results of the models. (figure 3 a)). These profiles are yielded by CLISTE at different time points after the break-down. The corresponding $j$ profiles are shown in figure 10. They are respectively, flat, peaked and with off-axis maximum. It is important to note that such a large variation of $q$ profiles from CLISTE does not occur for every discharge at the beginning of a current ramp. It is most likely dominated by the uncertainties of the equilibrium reconstruction.

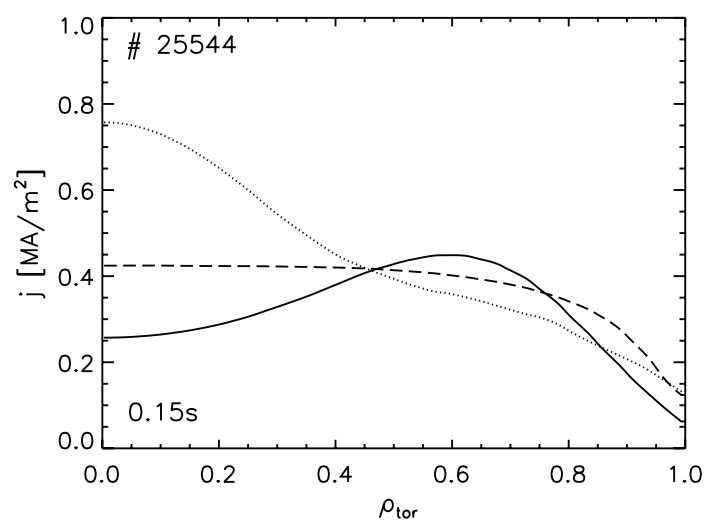

Figure 10. $j$ profiles at the beginning of the simulation resulting from different initial $q$ profiles figure 3 .

The resulting calculated $T_{\mathrm{e}}$ profiles for the three cases are illustrated in figure 11 at three different time points. The $T_{\mathrm{e}}$ profiles resulting from the Coppi-Tang and H98/2 models are more strongly affected by the initial conditions than the other three, see panel a. Furthermore, for these two models the effect of the initial conditions is still clearly visible at $0.55 \mathrm{~s}$ (panel $\mathrm{b}$ ), while the other three models exhibit almost no difference induced by the initial conditions at this time point. The Coppi-Tang model is still affected in the central part at the end of the current ramp. In both the Coppi-Tang and

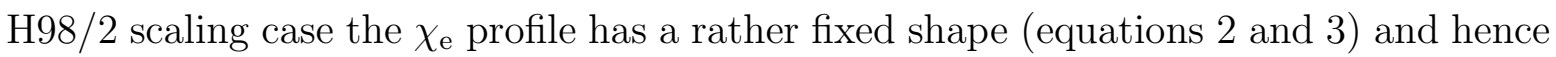
these models are strongly effected by the shape of the heating power density profile, $p_{\text {tot }}$, as will be discussed in the next section. In ohmic discharges the heating power density profile is mainly influenced by the current density distribution and hence the models strongly depending on $p_{\text {tot }}$ are strongly influence by the initial q-profile. Indeed, as shown by figure 11, both models yield $T_{\text {e }}$ profiles with off-axis maxima, in particular if the initial current density distribution is not centrally peaked. This is caused by the corresponding ohmic heating power profile with off-axis maximum. The influence of $p_{\text {tot }}$ on the other models, B/gB, CGM and Neo-Alcator, is marginal. These models depend additionally on $T_{\mathrm{e}}, \nabla T_{\mathrm{e}}$ or $q$ and hence are almost unaffected by the initial q profile. 

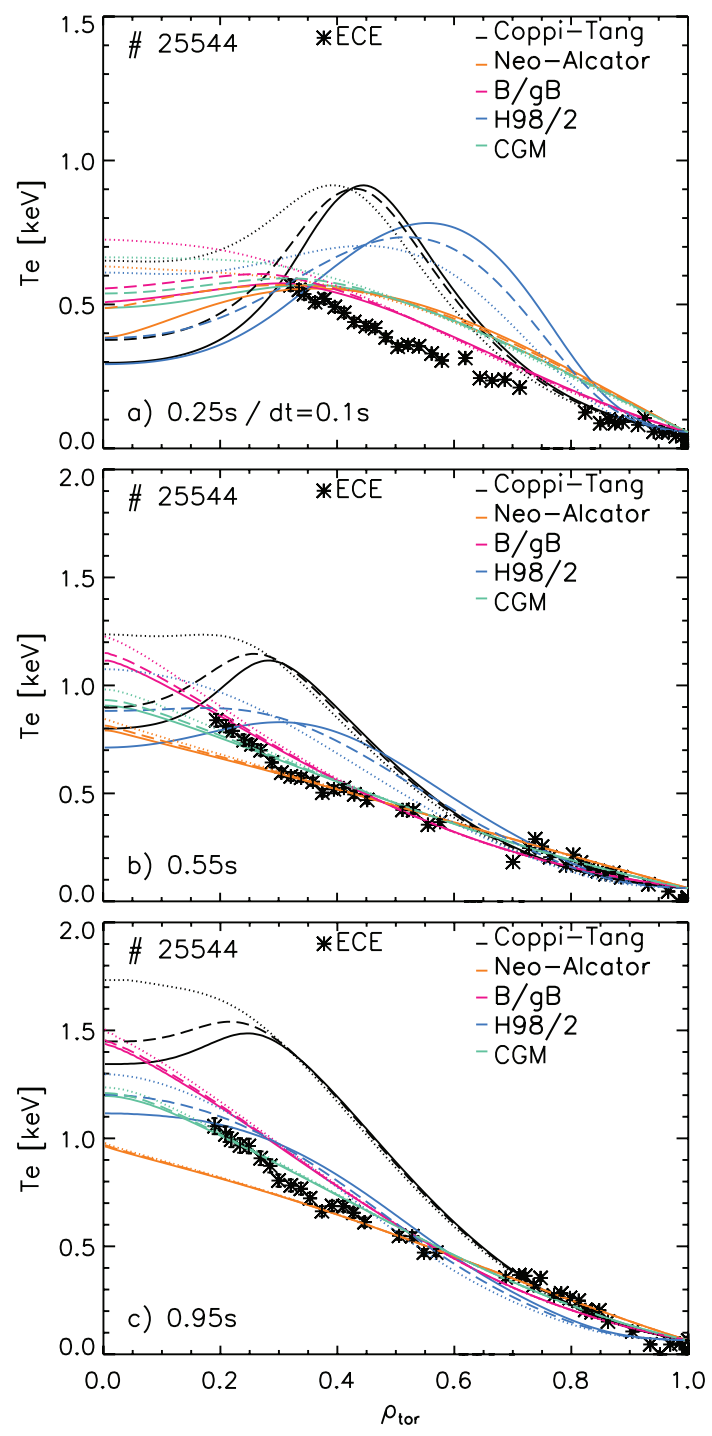

Figure 11. $T_{\mathrm{e}}$ profiles predicted by the models based on the different initial $q$ profiles according to figure $3 \mathrm{a}$ ) at the beginning, b) in the middle and c) at the end of the current ramp

However, also the influence of the initial q-profile on Coppi-Tang and the H98/2 scaling based model is relatively small compared to the general deviations from the experimental data. In summary, the choice of the initial $T_{\text {e }}$ profile does not effect the calculated $T_{\mathrm{e}}$ profiles after an adjustment time of about $100 \mathrm{~ms}$, and the affect of the initial q profile is also small for all models even if the influence on two model lasts over a fraction of the ramp time. 


\section{Results of the transport simulations}

For the validation of the models, the ramp-up phase of several discharges have been investigated which can be divided into three groups: ohmically heated with boronised and non-boronised wall, as well as non-boronised with ECRH. This provides the possibility to analyse the ability of the models to reproduce different experimental $T_{\mathrm{e}}$ profiles and to investigate the reaction of the models to various conditions in ASDEX Upgrade. The investigated discharges have also different combinations of flat-top density, magnetic field and plasma current, but the current ramp rate is always the same. As will be shown below, each group of current ramps exhibits a typical experimental $T_{\mathrm{e}}$ profile shape. Therefore each category of discharges sets the $\chi_{\mathrm{e}}$ models under different constraints.
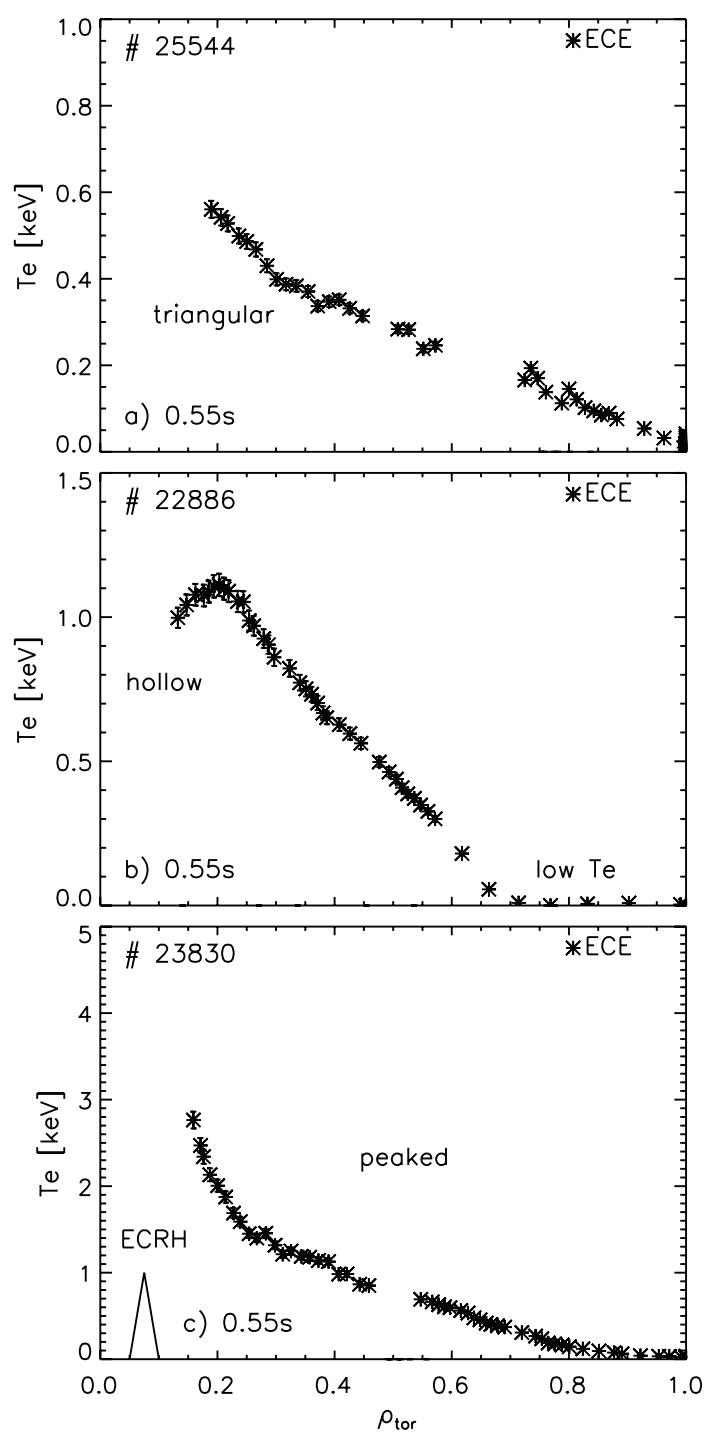

Figure 12. Examples for typical $T_{\text {e }}$ profiles under different conditions: a) boronised and ohmic, b) non-boronised and ohmic and c) ECR heated

The three typical experimental $T_{\mathrm{e}}$ profiles are shown in figure 12 . The profiles of 
ohmic current ramps in a boronised machine are "triangular", see fig. 12 a) and develop in time keeping this shape. The profiles of discharges with a non-boronised wall, with Ohmic heating only, are hollow in the centre inside $\rho<0.2\left(T_{\mathrm{e}}\right.$ deacrease of about $20 \%$ ) and exhibit a flat region with $T_{\mathrm{e}}<50 \mathrm{eV}$ near the edge due to radiation in the first part of the ramp, see fig. $12 \mathrm{~b}$ ). However, towards the end of the current ramp the flat $T_{\mathrm{e}}$ region near the edge vanishes due to the increase of the heating power and decrease of the radiation losses. Current ramps with ECRH deposited in the centre are characterised by strongly peaked electron temperature profiles, fig. 12 c).

These strong variations in the shape of the electron temperature profiles are partly reproduced by the models. In the following one exemplary discharge for each type of the three current ramps is shown and the experimental profiles are compared to the modelling results.

\subsection{Ohmic current ramp-up phases}

a) Boronised wall We first analyse current ramp-up phases performed with boronised wall. The experimental $T_{\mathrm{e}}$ profiles of these discharges are triangular. The ratio of core radiated power, $P_{\text {rad }}$, to the total heating power, $P_{\text {tot }}$, is lower than in the non-boronised cases and increases slightly during the ramp from 0.2 to 0.4 . The experimental lineaveraged $n_{\mathrm{e}}$ is increased by gas puffing during the current ramp. The variations in density during the ramp-up are limited by operational boundaries and the density ramp is similar for the discharges we investigated. The density profiles are rather flat during the whole ramp. The experimental $Z_{\text {eff }}$ at $\rho_{\text {pol }}=0.9$ decreases during the current ramp from values around six to almost 1 , whereas the central $Z_{\text {eff }}$ is around 1 during the whole ramp. As already discussed in section 4.2, different initial $q$ profiles can be chosen. This is also the case for the discharge presented in this section. The detailed analysis discussed here is based on a hollow q profile which yields the best calculated $T_{\mathrm{e}}$ profiles and we refer the reader to the previous section (figure 11) for the influence of the initial condition. For $T_{\mathrm{e}}$ and $T_{\mathrm{i}}$ the same time-dependent boundary condition is used: The temperature at the edge is set to be the experimental $T_{\mathrm{e}}$ at the separatrix. The magnetic field $B_{\text {tor }}$ of the discharge discussed below is $2.5 \mathrm{~T}$, the plasma current $I_{\mathrm{p}}$ is $0.8 \mathrm{MA}$ and $q_{95}$ is 5.4 at the end of the current ramp. The experimental radiation power density $\left(p_{\text {rad }}\right)$ profiles are provided by a deconvolution of the line integrated bolometric measurements with the assumption of a constant radiation emissivity on the flux surfaces [26]. The calculated ohmic heating power density $p_{\text {oh }}$ and the experimental $p_{\text {rad }}$ profiles of this discharge are shown in figure 13 for three time points.

During almost the whole current ramp $p_{\text {oh }}$ is higher than $p_{\text {rad }}$ over the whole radius. At the beginning of the ramp (figure 13 a)) the $p_{\text {oh }}$ profiles of all models are hollow due to the initial current density distribution. During the current ramp they are getting more and more peaked. In these discharges $p_{\text {rad }}$ near the centre decreases in time, while $p_{\text {rad }}$ at the edge increases (figure 13).

The hollow $p_{\text {oh }}$ profile and the central high radiation power near the centre at the 
beginning of the ramp result in a strongly hollow total heating power density profile, $p_{\text {tot }}$. During the current ramp the $p_{\text {tot }}$ profiles peak gradually due to the decreasing radiation losses and the evolving current diffusion towards peaked $j$ profiles.
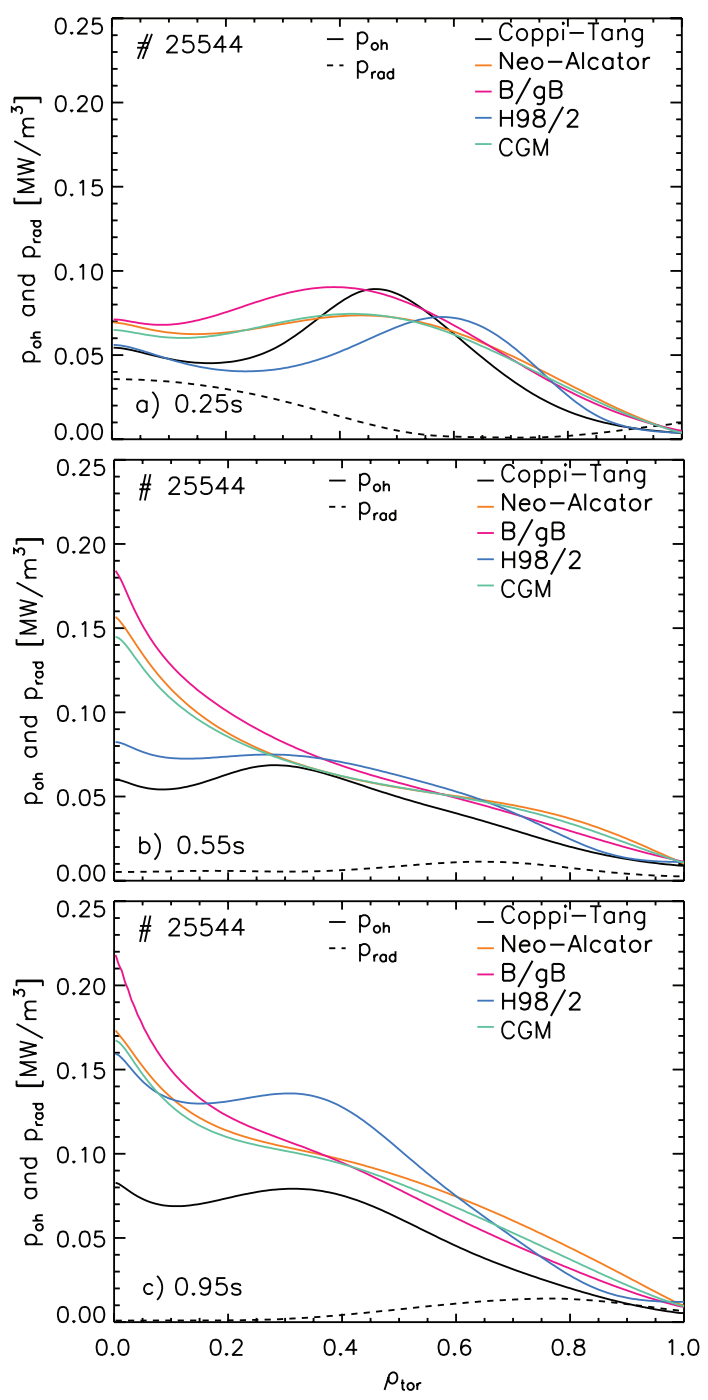

Figure 13. Calculated ohmic $\left(p_{\text {oh }}\right)$ and experimental radiation $\left(p_{\text {rad }}\right)$ power density profiles of \#25544 for the $\chi_{\mathrm{e}}$ models at three time points during the current ramp

The modelled and experimental $T_{\mathrm{e}}$ profiles at the same three time points during the current ramp are shown in figure 14 a)-c). In figure $14 \mathrm{~d}$ )-f) an additional assessment of the profiles quality is shown. In this quantitative analysis the ratio of the experimental $T_{\mathrm{e}}$ to the modelled $T_{\mathrm{e}}$ at the investigated time points is calculated at three or four radii which are chosen depending on the available radial location of experimental data. From this the deviation of the modelled profiles from the experimental one can be qualitatively assessed while also in some cases statements on the quality of the profile shape can be made. This analysis is provided in the same way for the modelling results in the next sub-sections.

At the beginning of the ramp the H98/2 scaling-based model and Coppi-Tang yield 

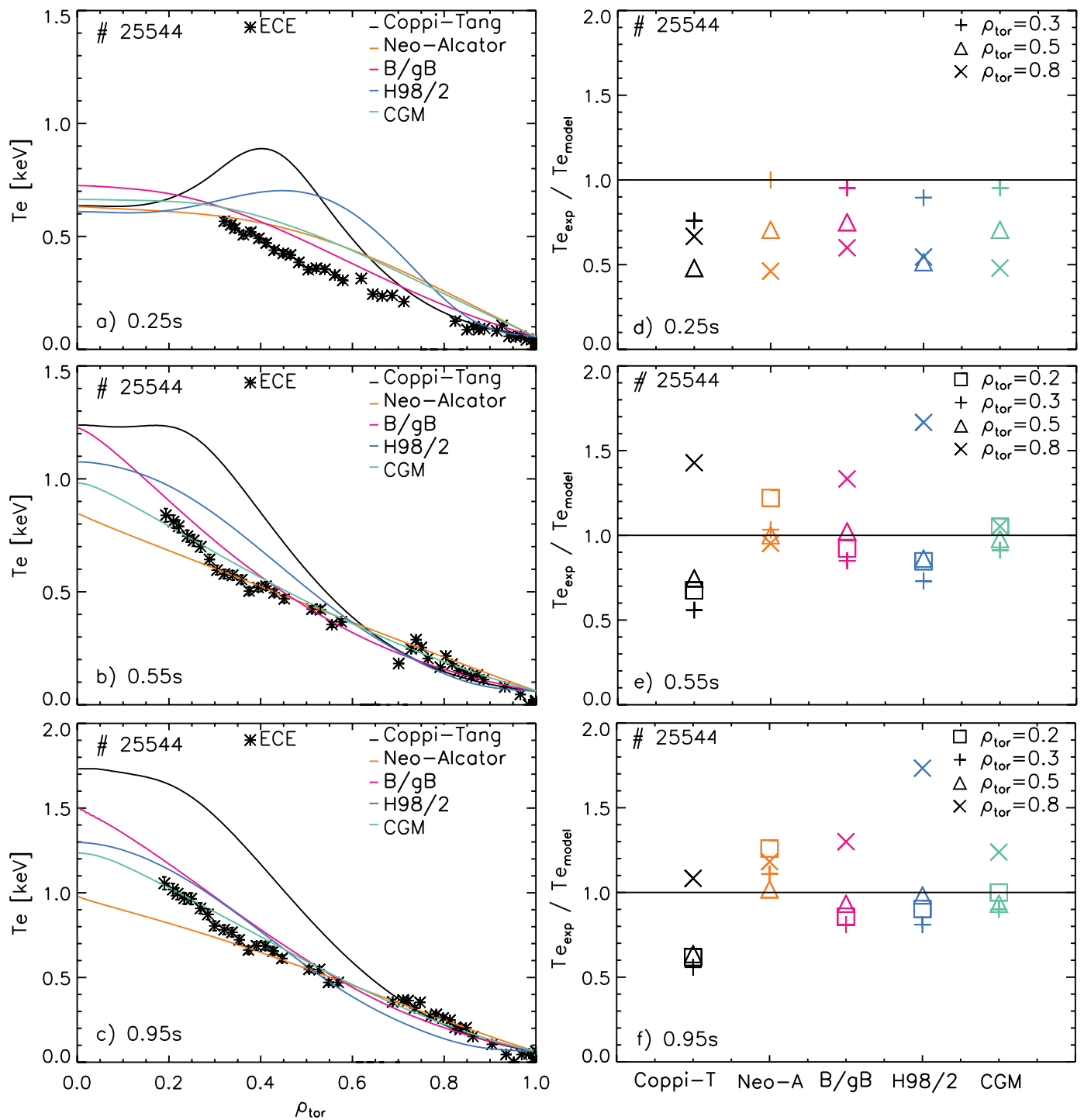

Figure 14. Comparison of modelled and experimental $T_{\mathrm{e}}$ profiles of a boronised discharge a) at the beginning $(0.25 \mathrm{~s}), \mathrm{b})$ in the middle $(0.55 \mathrm{~s})$ and $\mathrm{c})$ at the end $(0.95 \mathrm{~s})$ of the ramp-up phase. In panel d)-f) the ratio of the experimental $T_{\mathrm{e}}$ to the modelled $T_{\mathrm{e}}$ for each model is shown at different radii for every investigated time point to asses the profile quality. The different models are colour coded.

$T_{\text {e }}$ profiles with off-axis maxima and overestimate the experimental temperature between $\rho_{\text {tor }}=0.2-0.6$. The resulting $T_{\text {e }}$ profiles of these models are dominated by the $p_{\text {tot }}$ profiles due to their fixed $\chi_{\mathrm{e}}$ shape. Hence these two models result in $T_{\mathrm{e}}$ profiles with off-axis maxima due to the $p_{\text {tot }}$ profile.

The other three $\chi_{\mathrm{e}}$ models depend on $T_{\mathrm{e}}, \nabla T_{\mathrm{e}}$ or $q$ and hence the current density can be redistributed towards the centre which leads to a peaked $T_{\mathrm{e}}$ profile despite the fact that $p_{\text {tot }}$ profiles with off-axis maxima.

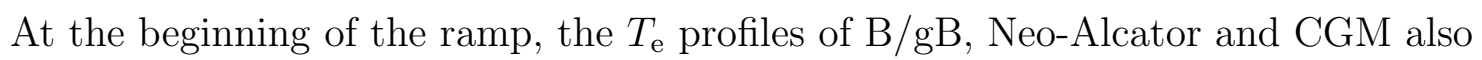
overestimate $T_{\mathrm{e}}$ around mid-radius, but less than the H98/2 and the Coppi-Tang models, see also figure $14 \mathrm{~d}$ ). 
All modelled $T_{\mathrm{e}}$ profiles are similar in the core region and in the same range as the extrapolation of the experimental data.

After one third of the current ramp phase, panels b, c and e, f of fig.14, B/gB, CGM, and especially Neo-Alcator for $\rho_{\text {tor }}>0.3$ yield $T_{\mathrm{e}}$ profiles which are in good agreement with the measured data. Only the Neo-Alcator model underestimates $T_{\mathrm{e}}$ towards the core for $\rho_{\text {tor }}<0.3$.

The off-axis maxima of the Coppi-Tang and H98/2 models disappear gradually during the current ramp, due to more and more centrally peaked $p_{\text {tot }}$ profiles caused by the decreasing radiation power density in the central region and a slow redistribution of the current density towards the core.

Now the Coppi-Tang model overestimates $T_{\mathrm{e}}$ over a large part of the radius until the end of the ramp, whereas the H98/2 scaling-based model yields $T_{\mathrm{e}}$ values comparable to the experimental one but with a curvature of the profile in the region $\rho_{\text {tor }}>0.5$. All models underestimate $T_{\mathrm{e}}$ towards the edge at $\rho_{\text {tor }}>0.8$. Overall the $\mathrm{B} / \mathrm{gB}$ model and CGM result in the best $T_{\mathrm{e}}$ profiles for discharges with boronised wall, when additionally considering the trend of the exp. data towards the core and the profile shaping.

b) Non-boronised wall The second group of discharges illustrated here were performed in a non-boronised machine with the tungsten wall. In these discharges the ratio $P_{\text {rad }} / P_{\text {tot }}$ is higher compared to the boronised cases. It increases from 0.4 up to 0.7 until $0.8 \mathrm{~s}$ and decreases afterwards down to reach 0.45 at the end of the current ramp. The experimental $T_{\mathrm{e}}$ profiles of these current ramps tend to be very flat or hollow in the central region, but the most striking feature is the very low values of $T_{\mathrm{e}}$ (only a few $10 \mathrm{eV}$ ) for $\rho_{\text {tor }}>0.6$, as shown in figure 15 . The region of low $T_{\mathrm{e}}$ at the edge is correlated with the existence of MHD activity near the edge which disappear at around $0.72 \mathrm{~s}$. Additionally high radiation losses at the edge and the core are observed. As the current builds up with time, the radiation at the edge decreases gradually and the low $T_{\mathrm{e}}$ region in the edge shrinks and eventually disappears at $t \approx 0.76 \mathrm{~s}$. So radiation losses at the edge together with edge MHD activity might be the reason for the low $T_{\mathrm{e}}$ in this region. The experimental $Z_{\text {eff }}$ and $n_{\mathrm{e}}$ profiles also differ from the profiles in a boronised machine. The $n_{\mathrm{e}}$ profiles are more peaked and the edge values of the $Z_{\text {eff }}$ profile are higher. They decrease from $Z_{\text {eff, } \rho_{\text {tor }}=0.9} \simeq 10$ at the beginning of the current ramp to $Z_{\text {eff }, \rho_{\text {tor }}=0.9} \simeq 6$ at the beginning of the flat-top phase. Also $Z_{\text {eff }}$ in the centre decreases from 2.5 to 1 during the current ramp.

Despite numerous attempts with various radiation profiles, the edge region with very low and flat temperature could not be reproduced by any model. An adjusted experimental boundary condition was therefore implemented until $\sim 0.76 \mathrm{~s}$ to take the very low $T_{\mathrm{e}}$ in the edge region into account. With this boundary condition, $T_{\mathrm{e}}$ is calculated with the $\chi_{\mathrm{e}}$ models for regions where the experimental $T_{\mathrm{e}}$ is higher than $50 \mathrm{eV}$, this means for $\rho_{\text {tor }}<\rho_{\text {tor }}(50 \mathrm{eV})$. For the edge region, where the experimental $T_{\mathrm{e}}$ is lower than $50 \mathrm{eV}$, a fit to the experimental data is used. The flat $T_{e}$ region disappears at $0.76 \mathrm{~s}$ and the boundary condition for $T_{\mathrm{e}}$ is then set to $70 \mathrm{eV}$ at $\rho_{\text {tor }}=1$ for the remaining current 
ramp. For $T_{\mathrm{i}}$ the same boundary condition is used.

All q profiles yielded by CLISTE from 0.05 to $0.2 \mathrm{~s}$ are hollow for this discharge. Therefore, in the following simulations a hollow initial q-profile is used, although this does not yield the best calculated $T_{\mathrm{e}}$ profiles. In figure $15 \mathrm{a}$ )-c), the $T_{\mathrm{e}}$ profiles of the ramp-up phase of a discharge performed in a non-boronised machine are shown for three time points during the current ramp (at the flat-top: $B_{\text {tor }}=2.1 \mathrm{~T}, I_{\mathrm{p}}=0.8 \mathrm{MA}, q_{95}=4.4$ ). A quantitative analysis is shown in figure $15 \mathrm{~d}$ )-f), where the quality of the profiles is assessed at different radii at three time points during the discharge.

The calculated $T_{\mathrm{e}}$ profiles without adjusted boundary condition (dashed lines) are also shown for comparison but not discussed in detail. Without adjusted boundary condition none of the models is able to reproduce the experimental $T_{\mathrm{e}}$ profiles, particularly at the edge.

By excluding the region of low $T_{\mathrm{e}}$ at the edge from the modelling, the experimental $T_{\text {e }}$ profiles which have to be simulated are almost triangular with only a flat or hollow region near the centre. At the beginning of the ramp, the $T_{\mathrm{e}}$ profile shape calculated with Coppi-Tang is similar to that of the experimental profile but the value overestimated over the whole radius. The profiles of the four other models are similar around mid-radius, they all overestimate $T_{\mathrm{e}}$ for $\rho_{\text {tor }}=0.3-0.7$ at the beginning of the ramp. Towards the centre $\rho_{\text {tor }}<0.2$, CGM and B/gB fit well, whereas the H98/2 and Neo-Alcator models predict too low $T_{\mathrm{e}}$. During the remaining current ramp the $T_{e}$ profiles are flat in the central region which is due to the sawtooth activity. Indeed, in the non-boronised cases, $q$ falls below unity very early in the current ramp because of the low edge temperature which forces a quite peaked current profile. This effect is well reproduced by the simulations and seen in the $q$ profiles yielded by the CLISTE equilibrium in which the central $q$ drops below 1 already at $0.4 \mathrm{~s}$. In the magnetic data a $1 / 1$ mode is detected, which appears at around $0.5 \mathrm{~s}$ and thenceforward is present during the rest of the current ramp. This again confirms the existence of the $q=1$ surface early in the current ramp such as yielded by CLISTE and ASTRA. With the aid of this mode at around $0.95 \mathrm{~s}$ the location of the $\mathrm{q}=1$ surface could be determined at $\rho_{\text {tor }}=0.25$. At this time point the calculation in ASTRA predicts the $q=1$ surface to be at $\rho_{\text {tor }}=0.4$ which is further outside. This suggests differences in calculated and experimental current diffusion but the general trend is well reproduced in the simulation. Consequently, in this part of the current ramp the central region is best calculated by Coppi-Tang which includes the effect of the sawteeth. Also for $\rho_{\text {tor }}>0.2$ this model agrees well with the experimental $T_{\mathrm{e}}$, see figure $15 \mathrm{e}$ ) and $\mathrm{f}$ ). The $\mathrm{B} / \mathrm{gB}$ model and the CGM also predict profiles which fit well for $\rho_{\text {tor }}>0.2$ whereas they deviate from the hollow experimental data in the central region. Neo-Alcator and the H98/2 scaling-based model underestimate the experimental $T_{\mathrm{e}}$ over the whole radius and are also not able to model the trend of a hollow central $T_{\mathrm{e}}$ profile. At the end of the current ramp the flat region at the edge vanishes and all models, except the H98/2 scaling model, agree well with the experimental data for $\rho_{\text {tor }}>0.8$. 

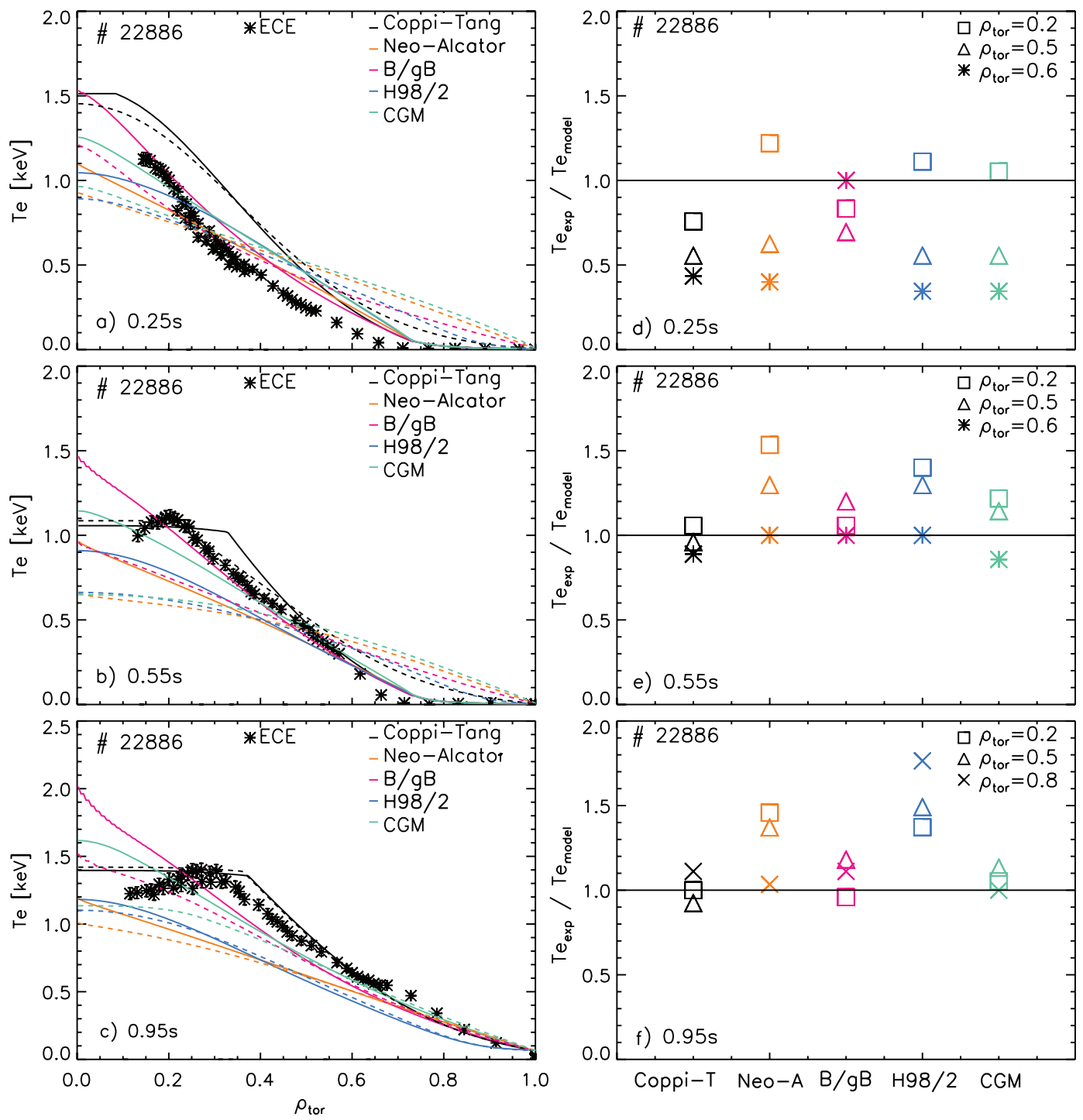

Figure 15. Comparison of modelled and experimental $T_{\mathrm{e}}$ profiles of a non-boronised, ohmic discharge a) at the beginning $(0.25 \mathrm{~s}), \mathrm{b})$ in the middle $(0.55 \mathrm{~s})$ and $\mathrm{c})$ at the end $(0.95 \mathrm{~s})$ of the ramp-up phase. The dashed profiles are just for comparison and calculated without using the adapted boundary condition. In panel d)-f) the ratio of the experimental $T_{\mathrm{e}}$ to the modelled $T_{\mathrm{e}}$ for each model is shown at different radii for every investigated time point to asses the profile quality. The different models are colour coded.

\subsection{ECRH assisted current ramps}

In addition to the Ohmic current ramps, ECRH assisted current ramps have also been performed in the non-boronised machine whose modelling results are presented in this section. For this analysis a discharge with $B_{\text {tor }}=2.5 \mathrm{~T}, I_{\mathrm{p}}=0.8 \mathrm{MA}$ and $q_{95}=5.0$ in the flat-top and moderate heating power is discussed. The ECRH power is deposited near the centre with a narrow profile. The ECRH power profile is calculated with the code TORBEAM [35], which yields a deposition width of about $1 \mathrm{~cm}$. At $0.3 \mathrm{~s}$ the ECRH is turned on with $0.65 \mathrm{MW}$ and at $0.9 \mathrm{~s}$ the heating power is increased to $1.4 \mathrm{MW}$. As this 
discharge was performed in a non-boronised machine, at the beginning of the current ramp the ratio of $P_{\text {rad }} / P_{\text {tot }}$ is very high. However, it decreases down to about 0.45 shortly after the ECRH was turned on. In figure 16, the time evolution of the heating power, Ohmic and ECRH, and the radiated power are shown.

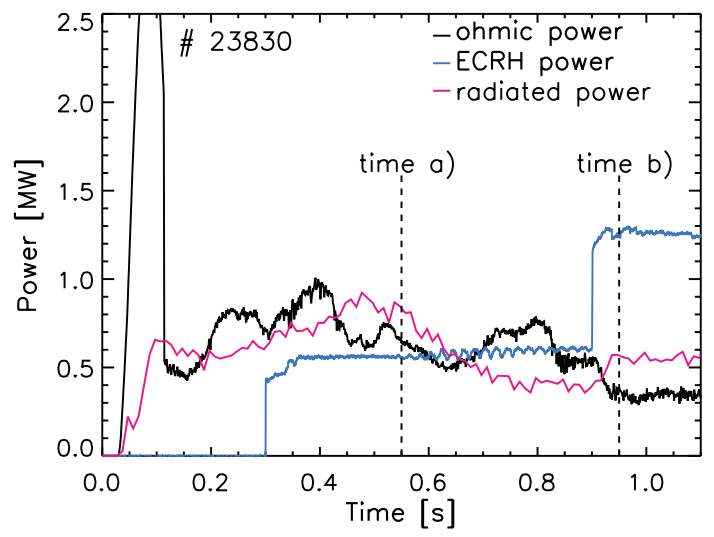

Figure 16. Time evolution of the ohmic heating power and the radiated power of a discharge in a non boronised machine with additionally applied ECRH power. The $\mathrm{ECRH}$ power is increased stepwise from $0.65 \mathrm{MW}$ at $0.3 \mathrm{~s}$ to $1.4 \mathrm{MW}$ at $0.9 \mathrm{~s}$.

Before the ECRH is switched on, the $n_{\mathrm{e}}$ and $Z_{\text {eff }}$ profiles are similar to the profiles of ohmic discharges in a non-boronised machine discussed above and also here the used initial q-profile is hollow. The experimental conditions and $T_{\mathrm{e}}$ profiles, and hence the results of the models at the beginning of the ramp until the ECRH is turned on are similar to those discussed in section $5.1 \mathrm{~b}$ ). Therefore, in this section, we focus on the phase with ECRH in the ramp-up. The profiles in the ohmic ramp-up without ECRH are not shown.

The heating power density profiles are dominated by the ECRH, which leads to centrally peaked experimental $T_{\text {e }}$ profiles as shown in figure 17 a) and b). Especially at the radial position of the ECRH deposition the ohmic power density is small, almost negligible, compared to the ECRH power density. The modelling of this discharge, with the local deposited heating power and the resulting peaked profiles, is a selective test for the models. For this discharge a time-dependent boundary condition for $T_{\mathrm{e}}$ and $T_{\mathrm{i}}$ is also used. However, in contrast to the previous cases, it cannot be taken from the ECE due to the shine-through of the measurements which occurs due to the low density and high central $T_{\mathrm{e}}$ induced by the ECRH. The boundary condition was therefore set arbitrarily to increase during the ramp from $20 \mathrm{eV}$ up to $100 \mathrm{eV}$ at the separatrix. In figure $17 \mathrm{a}$ )-b), the predicted $T_{\mathrm{e}}$ profiles of the five models are compared to the experimental profiles for two time points with the different ECRH power levels. Also for this discharge a qualitative analysis is shown in figure $15 \mathrm{c}$ )-d), where the quality of the profiles is assessed at different radii at three time points during the discharge .

To evaluate the results of the models for this discharge, not only the $T_{\text {e }}$ profiles but also the $\chi_{\mathrm{e}}$ profiles (fig. 18) are analysed and compared to $\chi_{\mathrm{PB}}$ yielded by the power balance, based on the experimental $T_{\mathrm{e}}$ profiles. 

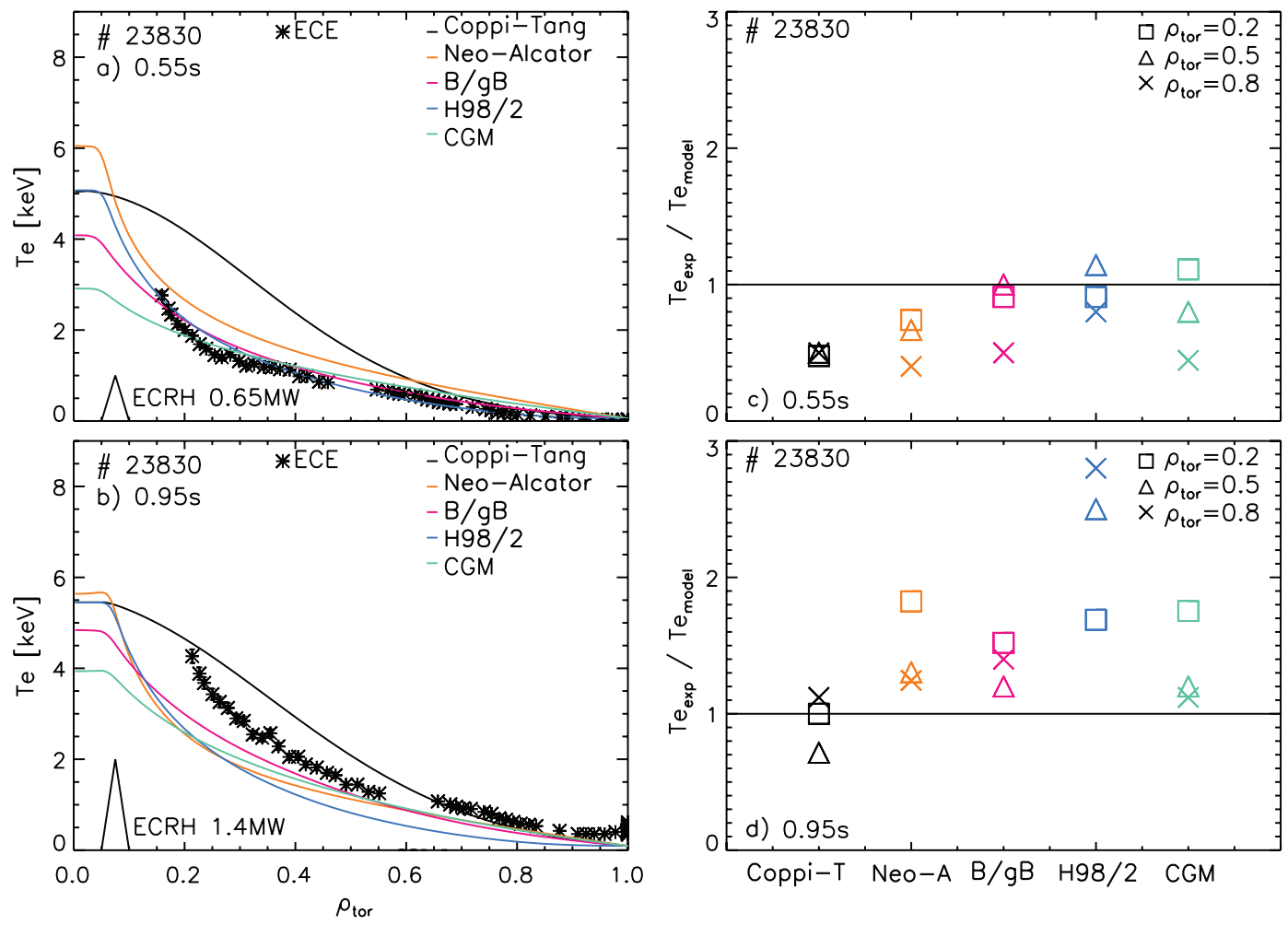

Figure 17. Comparison of modelled and experimental $T_{\mathrm{e}}$ profiles of a non-boronised discharge with two levels of ECRH. a) In the middle of the ramp $(0.55 \mathrm{~s})$ where $0.65 \mathrm{MW}$ ECRH are applied and b) at the end $(0.95 \mathrm{~s})$ of the ramp-up during the second phase of ECRH with an increased power of up to $1.4 \mathrm{MW}$. In panel c)-d) the ratio of the experimental $T_{\mathrm{e}}$ to the modelled $T_{\mathrm{e}}$ is shown for each model at different radii for every investigated time point to asses the profile quality. The different models are colour coded.

In order to obtain modelled $T_{\mathrm{e}}$ profiles close to the experimental centrally peaked $T_{\mathrm{e}}$ profiles, $\chi_{\mathrm{e}}$ must be high at the position of the deposited heating power, increase towards the core and towards the edge. Unfortunately no experimental $T_{\mathrm{e}}$ data are available inside $\rho_{\text {tor }}=0.2$ and additionally it is important to note here that the position and the amplitude of the $\chi_{\mathrm{PB}}$ maximum depend strongly on the location and width of the ECRH deposition profile. Therefore, the position and amplitude of the maximum of the $\chi_{\mathrm{PB}}$ profile is only used as a qualitative criterion.

In consequence the calculated $\chi_{\mathrm{PB}}$ profiles, which are illustrated in figure 18 , only serve as indication for the interpretation of the modelled $\chi_{\mathrm{e}}$ profiles.

The models react very differently to the locally deposited ECRH. This is more visible in the evolution of the $\chi_{\mathrm{e}}$ profiles of the five models than in the modelled $T_{\mathrm{e}}$ profiles.

Four models, Neo-Alcator, B/gB, CGM and H98/2, result in peaked $T_{\mathrm{e}}$ profiles comparable in shape to the experimental profile, though with different central $T_{\mathrm{e}}$ values. In contrast, the Coppi-Tang model predicts a differently shaped $T_{\mathrm{e}}$ profile and the $T_{\mathrm{e}}$ around $\rho_{\text {tor }}=0.2-0.4$ is two times higher than the experimental one during the first ECRH 


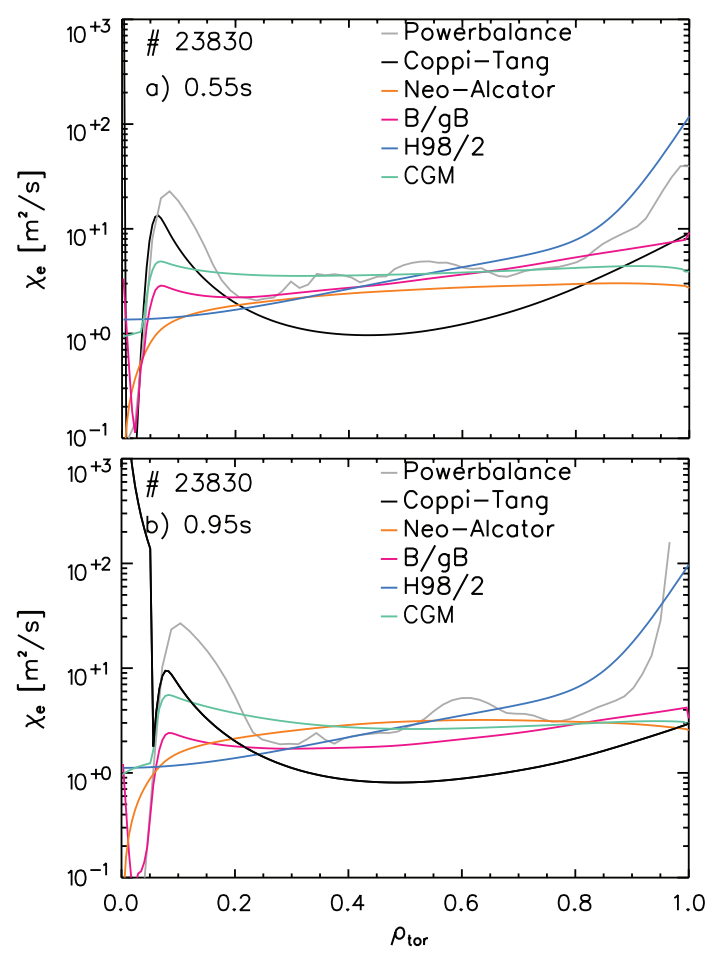

Figure 18. Comparison of the modelled $\chi_{\mathrm{e}}$ profiles with the $\chi_{\mathrm{e}}$ profile calculated with the power balance on the base of the experimental $T_{\mathrm{e}}$ profiles.

phase, figure $17 \mathrm{a}$ ) and c). All models predict a to high $T_{\mathrm{e}}$ towards the edge for $\rho_{\text {tor }}>0.8$. During the second heating phase the level of $T_{\mathrm{e}}$ yielded by Coppi-Tang is in the right range, but it should be underlined that the experimental $T_{e}$ increased.

Although the other four models yield similar $T_{\mathrm{e}}$ profiles, especially around $\rho_{\mathrm{tor}}=0.2$, which are in general too low $T_{\mathrm{e}}$, they all react differently to the locally deposited ECRH power, as emphasised by the $\chi_{\mathrm{e}}$ profiles plotted in figure 18 . The $\chi_{\mathrm{e}}$ profiles of the Neo-Alcator model and H98/2 scaling-based model are not able to adjust $\chi_{\mathrm{e}}$ in the required way. The Neo-Alcator model depends only on $1 / \mathrm{q}(\mathrm{r})$ and a local increase of $\chi_{\mathrm{e}}$, corresponding to the localised ECRH power, is not possible for this model, as can be seen in figure 18. Due to the moderate ECR heating power in this current ramp the inability of the Neo-Alcator model to handle the narrow deposited heating power is not reflected in the modelled $T_{\mathrm{e}}$ profiles. To increase $\chi_{\mathrm{e}}$ locally a dependence on the $T_{\mathrm{e}}, \nabla T_{\mathrm{e}}$ or $P_{\text {tot }}$ profile would be necessary.

The fixed parabolic shape of $\chi_{e}$ for the H98/2 scaling-based model does not allow any local increase of $\chi_{\mathrm{e}}$. A further disadvantage of this model is its sensitivity to the boundary condition. In fact, the principle of this model implies mainly that the plasma energy is adjusted to the level required by the condition $H_{98}=1 / 2$. Due to the volume effect, the plasma energy is dominated by the boundary condition of $T_{e}$. For instance, increasing $T_{\mathrm{e}}$ at the edge leads to a reduction of $T_{\mathrm{e}}$ in the central through an increase of $\chi_{\mathrm{e}}$. The effect is particularly strong in the presence of localised heating such as that of the ECRH. 
The heat diffusivity of the Coppi-Tang model increases locally in reaction to the local $P_{\mathrm{ECRH}}$ where at $\rho_{\text {tor }}=0.2$ it reaches values comparable to the power balance. Further out, it increases exponentially towards the edge, but remains too low in the region of $\rho_{\text {tor }}>0.2$. This leads to high $T_{\mathrm{e}}$ in the central region (see fig. $17 \mathrm{a}$ )) in the first ECRH phase and a differently shaped $T_{\text {e }}$ profile, meaning no peaked $T_{\text {e }}$ profiles. The CoppiTang model has also a weak feedback mechanism, which results from a dependence of this model only on the total heating power profile and not on $T_{\mathrm{e}}$.

The models B/gB and CGM depend directly on $T_{\mathrm{e}}$ and $\nabla T_{\mathrm{e}} / T_{\mathrm{e}}$ and, therefore, are able to react to the heating power by adjusting the $\chi_{\mathrm{e}}$ profiles locally. Compared to $\chi_{\mathrm{PB}}$ the $\chi_{\mathrm{e}}$ of both models have about the right shape but are slightly lower over the whole radius (figure 18). Then these two models not only result in $T_{\mathrm{e}}$ profiles, close in shape to the experimental $T_{\mathrm{e}}$ profiles, but also the $\chi_{\mathrm{e}}$ profiles meet the demands to handle ECRH. 


\section{Summary and Conclusion}

Five commonly used heat conductivity models have been applied to ASDEX Upgrade current ramps to investigate their ability to model these conditions. These models have been originally developed for L-mode flat-top conditions but are also used to model the current ramp. The five models, Coppi-Tang, Neo-Alcator, Bohm/gyroBohm (B/gB), a H98/2 scaling-based model and the "critical gradient model" (CGM), have all been developed to characterise low power discharges. To benchmark these models the calculated electron temperature profiles are compared to the experimental profiles. In this analysis the heat conductivity models are applied to ohmic and ECRH assisted ASDEX Upgrade current ramps-up with full tungsten coated inner wall. The three different types of ramp-up phases, investigated in this paper, ohmic boronised and nonboronised and ECRH assisted, result in quite different modelling conditions. Thus the heat conductivity models can be tested with regard to the modelling of differently shaped $T_{\text {e }}$ profiles (triangular, flat or hollow and peaked), the influence of different initial conditions and their response to different total heating power density profile. Different parameters are varied and hence provide a selective test based on the different conditions in the three investigated current ramp types.

The results of the ohmic current ramps strongly depend on the wall conditions, this means boronised or not boronised. Discharges performed with a non-boronised wall are dominated by strong radiation losses in the early ramp phase. Radiation losses at the edge lead to very low $T_{\mathrm{e}}$ there and hence peaked current density profiles. This, on the other hand leads, to early core MHD activity, i.e sawtooth crashes and 1/1 modes, and radiation losses in the centre. Hence the $T_{\mathrm{e}}$ profiles of non-boronised discharges are hollow with a flat, low $T_{\mathrm{e}}$ region towards the edge. To avoid the problem of low $T_{\mathrm{e}}$ at the edge for this type of current ramp an adjusted boundary condition had to be implemented.

In most of the previous analyses the quality of the models has been evaluated by comparing the calculated and experimental $T_{\text {e }}$ profile and additionally the time evolution of the internal inductance, due to its importance for the plasma stability and shaping. The internal inductance is a normalised volume averaged quantity and therefore strongly depending on the radial distribution of the $T_{\mathrm{e}}$ profiles. We performed a sensitivity study concerning this influence and showed that evaluating the quality of the $\chi_{\mathrm{e}}$ models by means of the internal inductance can lead to a misinterpretation. Therefore, in this study we evaluate the models only by comparing the calculated and experimental $T_{\text {e }}$ profile. In contrast to flat-top simulation, initial conditions are important for time-evolving plasma properties and profiles in the current ramp. The sensitivity of the results to the initial conditions for the electron temperature and the current density have been investigated. It turns out that the influence of the initial $T_{\mathrm{e}}$ profile is negligible for all models whereas the influence of the initial current density distribution strongly depends on the model, but is small compared to the large deviation between some of the modelling results and the experimental data. 
Applied to the three types of ASDEX Upgrade current ramps the five $\chi_{\mathrm{e}}$ models yield very different results due to their different physics properties.

The two models, Coppi-Tang and the H98/2 scaling model, are characterised by a rather fixed shape of the $\chi_{\mathrm{e}}$ profile.

The shape of the $\chi_{\mathrm{e}}$ profile of the Coppi-Tang model is, on the one hand, influenced by the $p_{\text {tot }}(\Phi)$ profile but additionally depends on an exponential shaping function. In ohmically heated plasmas the $\chi_{\mathrm{e}}$ shape is dominated by the exponential function because the $p_{\text {oh }}$ profile is rather smooth and hence $\chi_{\mathrm{e}}$ rather fixed. Under these conditions, the modelled $T_{\mathrm{e}}$ profile is dominated by the total $p_{\text {tot }}$ profile because the shape of $\chi_{\mathrm{e}}$ can not be adapted which leads to the fact that the results are strongly influenced by the initial current density. Consequently, this model yields hollow $T_{\mathrm{e}}$ profiles in the case of a boronised ramp-up with high radiation losses near the core, whereas the experimental $T_{\mathrm{e}}$ profiles are triangular. In general this model tends to calculate $T_{\mathrm{e}}$ profiles which are flat towards the core also without using a possible implementation which takes sawtooth crashes into account. This leads to the best result when modelling nonboronised discharges with hollow experimental $T_{\mathrm{e}}$ profiles. In the case of ECRH during the current ramp the $\chi_{\mathrm{e}}$ profile is additionally influenced by the total heating power profile. This makes it possible to adapt $\chi_{\mathrm{e}}$ locally independent from the $p_{\text {tot }}$ profile and redistribute the additional heating power radially. Nevertheless Coppi-Tang results in flat $T_{\mathrm{e}}$ profile towards the core and hence can not reproduce the peaked experimental profiles.

The modelling results of the H98/2 scaling model are even more fixed than the one of Coppi-Tang. The H98/2 scaling model used in this analysis has a fixed parabolic shape, as often used in modelling studies before. Consequently, no local adjustment of $\chi_{\mathrm{e}}$ is possible and the match to different conditions is limited. This fixed shape results in a strong dependence on the initial current density distribution through the influence of the total heating power profile.

In addition, this model has a strong dependence on the used boundary condition which strongly influences the level of the whole $T_{\text {e }}$ profile. The big disadvantage of the H98/2 scaling model and the Coppi-Tang model is the weak, i.e. no direct, dependence of the $\chi_{\mathrm{e}}$ profile on local gradients like $\nabla T_{\mathrm{e}}$.

The model with the simplest approach is the used Neo-Alcator model, which only depends on the $q$ profile and on $1 / n_{\mathrm{e}}$. But due to the dependence on the q-profile, which is fast evolving at the beginning of the current ramp, this model is not strongly influenced by the heating power profile and hence the initial current density distribution has only weak impact. In ohmic current ramps this simple model results in triangular profiles which characterise well the experimental $T_{\text {e }}$ profile of the boronised discharges and non-boronised with adapted boundary condition except for the core region. In ECRH assisted current ramps with moderate heating power this model results in the required peaked $T_{\mathrm{e}}$ profiles.

The B/gB and the CGM models both depend on $T_{\mathrm{e}}$ and $\nabla T_{\mathrm{e}} / T_{\mathrm{e}}$ which enables them to adapt the $\chi_{\mathrm{e}}$ locally and in particular avoid a direct coupling between heating power 
density and the $T_{\mathrm{e}}$ profiles even in ohmic discharges. Therefore the initial current density as well as the locally deposited ECRH power can be quickly redistributed. These two models do not result in flat or hollow $T_{\mathrm{e}}$ profiles and hence have problems to simulate $T_{\mathrm{e}}$ near the core of non-boronised current ramps. These models calculate peaked $T_{\mathrm{e}}$ profiles in a ECRH assisted current ramp and yield $\chi_{\mathrm{e}}$ profiles close to the shape of the $\chi_{\mathrm{e}}$ profile calculated with the power balance.

Concluding, Coppi-Tang is the best model when reproducing discharges with hollow $T_{\mathrm{e}}$ profiles like in the non-boronised case, but fails when modelling peaked or triangular profiles. Peaked and triangular $T_{\mathrm{e}}$ profiles can be better described by the other four transport models, Bohm/gyro-Bohm, CGM, H98/2 scaling model and Neo-Alcator. One main disadvantage of the, in this paper used, H98/2 scaling model is that it is very sensitive to the initial current density distribution and the boundary condition due to its fixed parabolic shape.

Similarly, the ability of the Neo-Alcator model to adjust to different discharge conditions is limited due to the lack of dependence on $T_{\mathrm{e}}$ and $\nabla T_{\mathrm{e}}$.

Therefore most flexible and reliable are transport models which depend on $T_{\mathrm{e}}$ and $\nabla T_{\mathrm{e}}$ because they allow for a decoupling of heating power density and the $T_{\text {e }}$ profiles. These models can adapt best to the different conditions in the current ramp. In general models whose results strongly depend on the initial conditions and profiles, can not be used to predict future device current ramps.

To improve the results of the models in general an accurate and reliable method to determine the initial current density distribution is needed due to the impact of this on some models. Even then these models whose results strongly depend on the initial conditions and profiles, are not useful to predict future device current ramps.

It is also important to include accurate radiation profiles to improve the results due to the influence of these on the total heating power profile and the difficulties to measure the distribution of the radiated power accurately. The implementation of an adjusted and accurate boundary condition is also important to obtain reliable results of the modelling. 
[1] A.C.C. Sips, T.A. Casper, E.J. Doyle, G. Giruzzi, Y. Gribov, J. Hobirk, G.M.D. Hogeweij, L.D. Horton, A.E. Hubbard, I. Hutchinson, S. Ide, A. Isayama, F. Imbeaux, G.L. Jackson, Y. Kamada, C. Kessel, F. Kochl, P. Lomas, X. Litaudon, T.C. Luce, E. Marmar, M. Mattei, I. Nunes, N. Oyama, V. Parail, A. Portone, G. Saibene, R. Sartori, J.K. Stober, T. Suzuki, S.M. Wolfe, the C-Mod team, the ASDEX Upgrade Team, the DIII-D team, and JET EFDA Contributors. Experimental studies of iter demonstration discharges. Nuclear Fusion, 49(8):085015, 2009.

[2] M. Mattei, M. Cavinato, G. Saibene, A. Portone, R. Albanese, G. Ambrosino, L. D. Horton, C. Kessel, F. Koechl, P. J. Lomas, I. Nunes, V. Parail, R. Sartori, A. C. C. Sips, and P. R. Thomas. Iter operational space for full plasma current h-mode operation. Fusion Engineering and Design, 84(2-6):300-304, 2009.

[3] F. Imbeaux, J. Citrin, J. Hobirk, G.M.D. Hogeweij, F. Kchl, V.M. Leonov, S. Miyamoto, Y. Nakamura, V. Parail, G. Pereverzev, A. Polevoi, I. Voitsekhovitch, V. Basiuk, R. Budny, T. Casper, J. Fereira, A. Fukuyama, J. Garcia, Y.V. Gribov, N. Hayashi, M. Honda, I.H. Hutchinson, G. Jackson, A.A. Kavin, C.E. Kessel, R.R. Khayrutdinov, C. Labate, X. Litaudon, P.J. Lomas, J. Lnnroth, T. Luce, V.E. Lukash, M. Mattei, D. Mikkelsen, I. Nunes, Y. Peysson, P. Politzer, M. Schneider, G. Sips, G. Tardini, S.M. Wolfe, V.E. Zhogolev, ASDEX Upgrade Team, C-Mod Team, DIII-D Team, JET-EFDA contributors, JT-60U Team, Tore Supra Team, contributors of the EU-ITM ITER Scenario Modelling group, ITPA Integrated Operation Scenarios group members, experts, ITPA Transport, Confinement group members, and experts. Current ramps in tokamaks: from present experiments to iter scenarios. Nuclear Fusion, 51(8):083026, 2011.

[4] E Fable, C Angioni, S Fietz, J Hobirk, G Pereverzev, and the Asdex Upgrade Team. Transport physics of asdex upgrade current ramps: experiment and theory. Journal of Physics: Conference Series, 260(1):012009, 2010.

[5] V. Parail, P. Belo, P. Boerner, X. Bonnin, G. Corrigan, D. Coster, J. Ferreira, A. Foster, L. Garzotti, G.M.D. Hogeweij, W. Houlberg, F. Imbeaux, J. Johner, F. Kochl, V. Kotov, L. Lauro-Taroni, X. Litaudon, J. Lonnroth, G. Pereverzev, Y. Peysson, G. Saibene, R. Sartori, M. Schneider, G. Sips, P. Strand, G. Tardini, M. Valovic, S. Wiesen, M. Wischmeier, R. Zagorski, JET EFDA contributors, and EU ITM Task Force. Integrated modelling of iter reference scenarios. Nuclear Fusion, 49(7):075030, 2009.

[6] G. L. Jackson, P. A. Politzer, D. A. Humphreys, T. A. Casper, A. W. Hyatt, J. A. Leuer, J. Lohr, T. C. Luce, M. A. Van Zeeland, and J. H. Yu. Understanding and predicting the dynamics of tokamak discharges during startup and rampdown. Phys. Plasmas, 17:1396-1404, 2010.

[7] I Voitsekhovitch, A C C Sips, B Alper, M Beurskens, I Coffey, J Conboy, T Gerbaud, C Giroud, T Johnson, F Kchl, E de la Luna, D C McDonald, I Pavlenko, G V Pereverzev, S Popovichev, A N Saveliev, G Sergienko, S Sharapov, M Stamp, and JET-EFDA Contributors. Modelling of the jet current ramp-up experiments and projection to iter. Plasma Physics and Controlled Fusion, 52(10):105011, 2010.

[8] R Goldston. Energie confinement scaling in tokamaks: Some implications of recent experiments with ohmic and string auxiliary heating. Plasma Physics and Controlled Fusion, 26(1A):87-103, 1984.

[9] ITER Physics Expert Group on Confinement and Transport and ITER Physics Expert Group on Confinement Modelling and Database, ITER Physics Basis Editors. Chapter 2: Plasma confinement and transport. Nuclear Fusion, 39(12):2175, 1999.

[10] S. C. Jardin, M. G. Bell, and N. Pomphrey. Tsc simulation of ohmic discharges in tftr. 33:371, 1993.

[11] M. Erba, T. Aniel, V. Basiuk, A. Becoulet, and X. Litaudon. Validation of a new mixed bohm/gyrobohm model for electron and ion heat transport against the iter, tore supra and start database dicharges. 38:1013-1028, 1998.

[12] V Parail, B Balet, Yu Baranov, B Fischer, G Huysmans, J Lingertat, R Sartori, F Soldner, E Springman, and A Taroni. Transport properties and predictive modelling of jet h-mode and 
optimized shear discharges. Plasma Physics and Controlled Fusion, 40(5):805, 1998.

[13] M. Erba, A. Cherubini, V. Parail, E. Springmann, and A. Taroni. Development of a non-local model for tokamak heat transport in l-mode, h-mode and transient regimes. 39:261-276, 1997.

[14] Taroni A., Erba M., Springmann E., and Tibone F. Global and local energy confinement properties of simple transport coefficients of the bohm type. Plasma Physics and Controlled Fusion, 36(10):1629-1646, 1994.

[15] F. Ryter, G. Tardini, F. De Luca, H.-U. Fahrbach, F. Imbeaux, A. Jacchia, K. K. Kirov, F. Leuterer, P. Mantica, A. G. Peeters, G. Pereverzev, W. Suttrop, and ASDEX Upgrade Team. Electron heat transport in ASDEX Upgrade: experiment and modelling. Nuclear Fusion, 43(11):1396-1404, 2003.

[16] F Imbeaux, F Ryter, and X Garbet. Modelling of ech modulation experiments in asdex upgrade with an empirical critical temperature gradient length transport model. Plasma Physics and Controlled Fusion, 43(11):1503, 2001.

[17] X. Garbet, P. Mantica, F. Ryter, G. Cordey, and F. Imbeaux. Profile stiffness and global confinement. 46:1351-1373, 2004.

[18] J. Weiland, A.B. JarmÉn, and H. Nordman. Diffusive particle and heat pinch effects in toroidal plasmas. Nuclear Fusion, 29(10):1810, 1989.

[19] A. G. Peeters, C. Angioni, M. Apostoliceanu, F. Jenko, F. Ryter, and the ASDEX Upgrade team. Linear gyrokinetic stability calculations of electron heat dominated plasmas in asdex upgrade. Physics of Plasmas, 12(2):022505, 2005.

[20] F. Ryter, C. Angioni, A. G. Peeters, F. Leuterer, H.-U. Fahrbach, W. Suttrop, and ASDEX Upgrade Team. Experimental study of trapped-electron-mode properties in tokamaks: Threshold and stabilization by collisions. Physical Review Letters, 95(8):085001-1, 2005.

[21] G. Pereverzev and P. N. Yushmanov. Astra automated system for transport analysis in tokamak. IPP Report, (5/98), February 2002.

[22] V. D. Zakharov, L. E.; Shafranov. volume 11, page 153. Reviews of Plasma Physics, Consultants Bureau: NY-London, 1986.

[23] P. J. Mc Carthy. Analytical solutions to the grad-shafranov equation for tokamak equilibrium with dissimilar source functions. Physics of Plasmas, 6(9):3554-3560, 1999.

[24] S. P. Hirshman, R. J. Hawryluk, and B. Birge. Nucl. Fusion-Letters v.17, 3:611, 1977.

[25] R. Fischer, C. J. Fuchs, B. Kurzan, W. Suttrop, E. Wolfrum, and ASDEX Upgrade Team. Integrated data analysis of profile diagnostics at asdex upgrade. Fusion Science and Technology, 58(2):675-684, 2010.

[26] R. Wunderlich, W. Schneider, and K. Lackner. Entfaltung von bolometersignalen am tokamak asdex upgrade. IPP Report, 1992.

[27] S K Rathgeber, R Fischer, S Fietz, J Hobirk, A Kallenbach, H Meister, T Pütterich, F Ryter, G Tardini, E Wolfrum, and the ASDEX Upgrade Team. Estimation of profiles of the effective ion charge at asdex upgrade with integrated data analysis. Plasma Physics and Controlled Fusion, 52(9):095008, 2010.

[28] C. S. Chang and F.L. Hinton. Effect of finite aspect ration on the neoclassical ion thermal conductivity in the banana regime. 25:1493, 1982.

[29] C. S. Chang and F.L. Hinton. Effect of impurity particles on the finite-aspect ratio neoclassical ion thermal conductivity in a tokamak. 29:3314, 1986.

[30] F. Imbeaux et al. in Fusion Energy 2010 (Proc. 23rd Int. Conf. Daejeon, 2010) (Vienna: IAEA) CD-ROM file ITR/P1-20. http://wwwnaweb.iaea.org/napc/physics/FEC/FEC2010/html/index.htm.

[31] D.J. Ward, A. Bondeson, and F. Hofmann. Pressure and inductance effects on the vertical stability of shaped tokamaks. Nuclear Fusion, 33(5):821, 1993.

[32] R Neu, V Bobkov, R Dux, J C Fuchs, O Gruber, A Herrmann, A Kallenbach, H Maier, M Mayer, T Pütterich, V Rohde, A C C Sips, J Stober, K Sugiyama, and ASDEX Upgrade Team. Ten years of w programme in asdex upgrade - challenges and conclusions. Phys. Scr., T138:014038 
(6pp), 2009.

[33] A. Kallenbach, R. Dux, M. Mayer, R. Neu, T. Pütterich, V. Bobkov, J.C. Fuchs, T. Eich, L. Giannone, O. Gruber, A. Herrmann, L.D. Horton, C.F. Maggi, H. Meister, H.W. Müller, V. Rohde, A. Sips, A. Stbler, J. Stober, and ASDEX Upgrade Team. Non-boronized compared with boronized operation of asdex upgrade with full-tungsten plasma facing components. Nuclear Fusion, 49(4):045007, 2009.

[34] R. Dux, V. Bobkov, A. Herrmann, A. Janzer, A. Kallenbach, R. Neu, M. Mayer, H.W. Müller, R. Pugno, T. Pütterich, V. Rohde, and A.C.C. Sips. Plasma-wall interaction and plasma behaviour in the non-boronised all tungsten asdex upgrade. Journal of Nuclear Materials, 390 - 391(0):858 - 863, 2009. Proceedings of the 18th International Conference on Plasma-Surface Interactions in Controlled Fusion Device http://www.sciencedirect.com/science/journal/00223115/390\#390\#390.

[35] E. Poli, A. G. Peeters, and G. V. Pereverzev. Torbeam, a beam tracing code for electron-cyclotron waves in tokamak plasmas. Computer Physics Communications, 136(1-2):90 - 104, 2001. 